\title{
EFFECTIVE INTERNATIONAL EXPANSION STRATEGIES OF DEVELOPING COUNTRIES: SOME PRELIMINARY EVIDENCE FROM THE TURKISH MANUFACTURE AND EXPORT OF TELEVISION SETS
}

\author{
*Tanses GÜLSOY \\ **Özlem ÖZKANLI \\ ****Richard LYNCH
}

*Beykent University, Istanbul, Turkey

**Ankara University, Ankara, Turkey ****Middlesex University, London, UK

\begin{abstract}
Over the last twenty years, Turkey has significantly increased its international trade in manufactured products. It is possible that this has been built on the basis of its lower labour costs compared with those of developed countries and the willingness of both Turkish national companies and Multi National Enterprises (MNEs) to invest in Turkey. While the basic evidence of Turkish trade development is clear, there has been only limited research on the business logic behind such growth, the reasons for choosing particular forms of expansion and the outcomes of such strategies at the present time for Turkish companies.

In order to explore these issues, we have undertaken a preliminary study of the Turkish manufacture and export of television set companies. Our findings show that companies have largely focused on European markets. We do not yet know the reasons for this but will investigate this in the next stage of our research. It is possible that our later research may provide some assistance both to individual companies and to Turkish national policy makers on future export development. However, our primary purpose will be to shed further light on the content and process by which developing countries pursue effective international expansion strategies.
\end{abstract}

Keywords:

Global strategy; Developing Country Strategy; Turkey Export Strategy; Turkish Television Set Manufacture

\section{INTRODUCTION}

Over the last twenty years, Turkey has significantly increased its international trade in manufactured products: for example, merchandise exports measured in US dollars grew 284 per cent between years 2000 and 2007 (UNCTAD 2008). There is some evidence to suggest that this has primarily been 
built on the basis of its lower labour costs compared with those of developed countries and the willingness of both Turkish national companies and Multi National Enterprises (MNEs) to invest in Turkey (Dunning and Lundan 2008).

We have chosen to focus on the Turkish electronics industry because it has had some success in recent years (TÜİK 2008a). The industry contains six sub-sectors: electronic components, consumer electronics, telecommunications equipment, computers, professional and industrial electronics equipment, and military electronics (DPT, 2007: 7). We have selected consumer electronics for our main research at this stage because it is the largest single sector within this category.

According to the Turkish State Planning Organization (DPT), the consumer electronics industry includes: all kinds of color televisions, audio devices, electronic scales, electronic calculators, video recorders, cash registers, audio/video cassettes, TV satellite receivers, and antenna stations (DPT, 2007: 121). However, the main driver of growth within consumer electronics has been television sets. We have therefore chosen at this stage in our research to focus on television manufacture, import and export. In 2007, Turkish television manufacturers commanded 55\% of the European Union cathode-ray tube TV market (Turkish Ministry of Industry and Trade, 2008). In 200712.6 million sets were manufactured (TÜiK, 2008a), and that number was projected to reach 21 million sets by 2010 (DPT, 2007: 144). Television exports alone accounted for 1.9\% of Turkey's total exports for 2007 , revealing the strategic importance of the industry for Turkey's economy.

While the basic evidence of Turkish trade development is clear, there has been only limited research on the business logic behind such growth, the reasons for choosing particular forms of expansion and the outcomes of such strategies at the present time for Turkish companies. In turn, this relates to fundamental questions in international business literature: How do industries in developing countries like Turkey, China and India compete and sustain their growth over time? What are the key components of such international expansion strategies? To what extent are joint ventures, licensing and other forms of expansion important?

At the present time, there is only limited evidence on these broad and complex questions. We can find very few research papers at present on Turkish international expansion strategies. The purpose of this paper is to begin to address this issue. The new contribution of this paper is therefore to find the evidence and consider its implications for effective international expansion strategies of developing countries.

Our study explores Turkish television set manufacturers that have entered the global consumer electronics market after their well-established rivals. The reasons for choosing this product category are explored later in this paper. We draw on historic data for the shifting fortunes of other companies in the international consumer electronics industry to provide some strategic perspective. We then consider the following issues: How important have Turkish companies become? What are their prime sources of their international business at the present time? Why have they chosen particular strategies? And with what outcomes?

Specifically, this paper seeks to begin to address the following research questions:

1. How has Turkish television set manufacture grown over the last ten years? And with what results both internationally and domestically?

2. What strategies have Turkish companies used in their expansion? Specifically, what has been the role of pricing and innovation? 
3. What are the managerial implications for effective international strategy development by developing countries?

We wish to emphasise that this paper represents a preliminary, exploratory study. We do not expect to obtain full answers to the above questions from this current paper.

\section{LITERATURE REVIEW AND RESEARCH FRAMEWORK}

Although the literature in international business research is extensive and continues to grow (Werner, 2002), Axinn and Matthyssens (2002) have argued that most theories on international strategies focus on explaining the behaviour of large firms from developed countries. They do not provide the same insights into the behaviour of firms from developing nations in the international market place. Given the emergence of companies from new international trading nations like India, China and Turkey, the authors claim that it is imperative to look at new empirical evidence, rather than rely on existing theories of international trade. Equally, Buckley (2002) commented that the entry of developing countries as major players in the global economy may give new impetus to such research. This is echoed by other scholars' calls for research into strategies for firms from emerging economies (Peng, 2005).

In view of the preliminary nature of the research developed in this paper, we have chosen to summarise the main strands of the literature in the table below. We make the assumption that readers will be broadly familiar with the relevant papers and focus on drawing out the main highlights.

With regard to our research framework, we have taken the view that the first stage in our research is to gather and review the existing data on Turkish business activity in our chosen sector. As a second stage, we would then expect to explore the reasons behind the data and thereby draw out some conclusions on effective international expansion by Turkish companies and its implications for effective international expansion strategy by developing countries. However, this latter part does not form part of this initial approach.

\begin{tabular}{|l|l|}
\hline Strategic issues & \multicolumn{1}{|c|}{ Some references } \\
\hline International business expansion & $\begin{array}{l}\text { Dunning and Lundan 2008, Werner 2002; Axinn and Matthysens } \\
\text { 2002; Buckley 2002, Johanson and Vahlne 1977 }\end{array}$ \\
\hline $\begin{array}{l}\text { Global strategy and related issues in } \\
\text { regional strategy }\end{array}$ & $\begin{array}{l}\text { Levitt, 1983; Douglas and Wind, 1987; Ghoshal, 1987; Yip, 1992; } \\
\text { Dunning 1993; Dunning 1995; Ghemawat and Gaidar, 2000; Ghe- } \\
\text { mawat 2003; Rugman and Verbeke 2003a, Rugman and Verbeke } \\
\text { 2003b }\end{array}$ \\
\hline $\begin{array}{l}\text { Turkish international strategy - in- } \\
\text { cluding routes to development }\end{array}$ & $\begin{array}{l}\text { Taylan and Aari 2008, Ates 2006, Çakir, 2004, Karabati and Tan } \\
\text { 2005 }\end{array}$ \\
\hline Strategic process & $\begin{array}{l}\text { Huff and Reger, 1987; Pettigrew, 1992; Papadakis and Barwise, } \\
1997 ; \text { Chakravarthy and White, 2002 - who identify four routes }\end{array}$ \\
\hline $\begin{array}{l}\text { Entry and expansion strategies of } \\
\text { MNEs }\end{array}$ & $\begin{array}{l}\text { Dunning and Lundan 2008, Delios and Henisz 2003, Forsgren } \\
\text { 2002, Bartlett and Ghoshal 1989, Doz et al 2001 }\end{array}$ \\
\hline $\begin{array}{l}\text { Company resource management - } \\
\text { international decisions and expansion }\end{array}$ & $\begin{array}{l}\text { Doz 1986; Ohmae, 1983; Doz and Prahalad, 1991; Barney, 1991; } \\
\text { Peteraf, 1993; Murtha et al 1998; Grant, 2002, Lynch 2008 }\end{array}$ \\
\hline
\end{tabular}




\section{RESEARCH METHOD}

At this early stage in our research, we have taken an eclectic approach to the gathering of data. We have been more concerned to see where the strands of information might lead us than to address specific hypotheses. Our approach has therefore been to examine the existing trade and company data in Turkey from an international development perspective.

The main data sources have been developed under the guidance and sponsorship of the Turkish Ministry of Industry and Trade (2008), the State Planning Organisation of Turkey DPT (2007), and the Istanbul Chamber of Industry (2001 and 2008). We have drawn heavily on these sources in our initial examination of the issues. However, some of this data has been summarised in reports prepared by the Turkish government and other official organisations and may therefore be subject to the opinions of those tasked with drawing up reports on Turkish international performance. To ensure that our data is accurate and not subject to interpretation bias, we have used the raw data from the relevant Turkish government offices in the compilation of our research rather than rely solely on the finished reports.

Initially we had some difficulty with the data from these sources but we have now resolved these problems. We are confident that our basic research information provides an adequate picture of the state of television production and international trade up to 2007. However, we would caution that the international trade downturn in 2009 is not reflected in our data set. Effective international development strategies may need to be reconsidered in this latter context over the period from 2010.

In addition to Turkish government official statistics, we have used the business press, personal contacts and other reports to develop as full a picture as possible at this stage of the Turkish television set industry, its role and strategies in international trade development.

\section{PRELIMINARY RESEARCH RESULTS}

\section{Brief History of Consumer Electronics in Turkey}

The Turkish electronics industry, considered Turkey's third biggest industry following textiles and construction, has had its beginnings in the early 1950s, in radio assembly manufacturing. This was followed by the manufacturing of telephone operating systems in the 1960s, and television manufacturing in 1968. In 1964, the government laid the groundwork for the establishment of a Turkish electronics industry by including a feasibility report in the First Five-Year Development Plan (Çakır, 2004: 4). In the second half of the 1960s, this time the start was given to the telecommunications industry. Therefore, mid-1960s can be said to have been critical years for the Turkish electronics industry.

The consumer electronics subindustry began to develop rapidly in the second half of the 1980s. Giving it fresh impetus was the beginning of investments in color broadcasting. Again during this decade the contribution of foreign direct investment made possible manufacturing in many different subindustries of electronics (Çakır, 2004: 5). Most Turkish companies began production through foreign license agreements, with the licensors originating from the Netherlands, Germany, Japan, USA, Canada, France, the UK, Denmark, and Italy (Sar1 and Taylan, 2008: 1). The liberalization policies of the 1980s translated into increased productivity for consumer electronics manufacturers, who, as a result, increased their exports significantly (Çakır, 2004: 5). Today the industry can be said to have reached a significant level of technical proficiency. 


\section{Turkey's Place in the World Television Set Industry}

World television sales were estimated to be around 107 billion dollars in 2007 (Turkish Ministry of Industry and Trade, 2008). Asian countries' share in total production seems to continue increasing while that of Western Europe and the US decreases even if slightly (Çakır, 2004: 7). Japan is the world leader in color television manufacturing, followed by China and South Korea (ibid.). In the world market for television sets the biggest shares belong to Samsung (14\%), LG (11\%), Philips (7\%), Sony (6\%), and TCL (6\%) (Turkish Ministry of Industry and Trade, 2008).

With exports of television sets estimated to be around 2.03 billion dollars in 2007 (TÜİK, 2008b), Turkey has a $1.9 \%$ share in world television sales. Market share in Europe is estimated to be $40 \%$ (Turkish Ministry of Industry and Trade, 2008).

Prior to 1970, television manufacturing was vertically integrated; today, however, companies utilize external suppliers more and outsource manufacturing and design of components and products (Karabatı and Tan, 2005). During the 1990s, Turkey appeared as a low-cost manufacturing destination (ibid.). While Turkish manufacturers usually work as OEMs for manufacturers in Europe, by engaging in serious investments, some of them have turned into ODMs in time (DPT, 2007: 126).

Turkey captured the position of leading manufacturer in Europe in 1997 (Çakır, 2004: 11) and continues to maintain that position. The bulk of Turkish TV exports is composed of cathode-ray tube television sets (Please see Tables 4 and 5). Consumer gravitation away from CRT sets towards larger screen LCD and plasma sets is expected to grow exponentially (DPT, 2007: 129), and this trend is apparent in major markets such as the US, where CRT sets have already ceded leadership to flat panels (DuBravac, 2007).

\section{Production and Sales}

Production of television sets in Turkey began in 1968, with a mere 1,532 sets (Çakır, 2004: 10). As television broadcasting spread in the 1970s, demand for television receivers rose. By 1971, production had increased nearly five-fold to 6,882 units (ibid.). Starting in 1972, imports decreased, and local manufacturers began to dominate the home market. Between 1968 and 1984 (the black-andwhite television era), 1977 stands out as the year when black-and-white TV set production reached its peak (683,848 units) (ibid.). In 1984 color broadcasting began across the country, as a result of which demand for color television receivers climbed steeply. Color television production began in 1983, and starting in 1990, all of the television sets produced were color TVs (Usta, 2002: 14).

Factory sales of television sets for 25 years, between 1983-2007, are given in Table 1. 
Table 1: Television production in Turkey, 1983-2007

\begin{tabular}{|c|c|c|}
\hline Year & Total Factory Salers (UNITS) & Rate of Growth Year on Year \\
\hline 1983 & 419.276 & \\
\hline 1984 & 819.001 & 0,95 \\
\hline 1985 & 1.016 .616 & 0,24 \\
\hline 1986 & 870.843 & $-0,14$ \\
\hline 1987 & 690.187 & $-0,21$ \\
\hline 1988 & 738.221 & 0,07 \\
\hline 1989 & 999.264 & 0,35 \\
\hline 1990 & 1.994 .621 & 1,00 \\
\hline 1991 & 2.567 .773 & 0,29 \\
\hline 1992 & 2.111 .089 & $-0,18$ \\
\hline 1993 & 1.921 .704 & $-0,09$ \\
\hline 1994 & 1.528 .255 & $-0,20$ \\
\hline 1995 & 1.859 .333 & 0,22 \\
\hline 1996 & 2.509 .712 & 0,35 \\
\hline 1997 & 4.657 .007 & 0,86 \\
\hline 1998 & 5.794 .771 & 0,24 \\
\hline 1999 & 6.941 .004 & 0,20 \\
\hline 2000 & 8.788 .294 & 0,27 \\
\hline 2001 & 8.025 .118 & $-0,09$ \\
\hline 2002 & 12.462 .924 & 0,55 \\
\hline 2003 & 15.035 .590 & 0,21 \\
\hline 2004 & 20.345 .757 & 0,35 \\
\hline 2005 & 20.790 .123 & 0,02 \\
\hline 2006 & 17.930 .371 & $-0,14$ \\
\hline 2007 & 12.591 .569 & $-0,30$ \\
\hline Total & 153.408 .423 & \\
\hline
\end{tabular}

Source: TÜIKK Turkish Statistics Institute, 2008a.

The production of television sets follows to some extent economic vicissitudes, shrinking in recession years and rebounding once the economy becomes stronger. The economic recession of 1994 caused the contraction of the entire consumer electronics output in Turkey by $50 \%$ and forced the industry to seek overseas markets (Istanbul Chamber of Industry, 2001: 34). Consequently, between 1995 and 1999 the share of exports in total consumer electronics output exceeded 50\% (ibid.).

Television set output, in the year 2000, increased by $27 \%$ compared to the previous year, reaching nearly 9 million sets (Please see Table 1). This was largely the result of the implementation of a fixed exchange rate policy, a relative decrease in interest rates, extension of consumer credit, and promotional campaigns, which stimulated demand (Çakır, 2004: 11). The growth trend, however, was disrupted with the severe recession experienced in 2001, and the industry's problems were further compounded with an anti-dumping investigation instigated by the EU in the year 2000 (Careful investigation of the costs of Turkish manufacturers later resulted in the dropping of anti-dumping charges against them). Consequently, output contracted by $9 \%$ from the previous year, and domestic sales fell by $44 \%$. The industry managed to survive the recession by emphasizing export sales, 
thanks to which production rebounded the following year with a robust $55 \%$ increase (Please see Table 2). Between the year 2000 and 2006, the share of television exports in total television production consistently topped $80 \%$, and frequently exceeded $90 \%$.

Table 2: Production, Exports, Sales, and Home Market Sales of TV Sets in Turkey, 1998-2007

\begin{tabular}{|c|c|c|c|c|c|c|}
\hline \multicolumn{7}{|c|}{ TELEVISION SET PRODUCTION, EXPORTS, AND HOME SALES, 1998-2007 } \\
\hline & Production (Units) & $\begin{array}{c}\text { Exports } \\
\text { (Units) }\end{array}$ & $\begin{array}{l}\text { Share of } \\
\text { Exports } \\
\text { in Pro- } \\
\text { duction }\end{array}$ & $\begin{array}{c}\text { Total TV Sales } \\
\text { (incl. both exports } \\
\text { and home sales of } \\
\text { domestically pro- } \\
\text { duced TV sets) } \\
\text { (Units) }\end{array}$ & $\begin{array}{c}\text { Home Mar- } \\
\text { ket Sales* } \\
\text { (Units) }\end{array}$ & $\begin{array}{c}\text { Share of } \\
\text { Home } \\
\text { Market } \\
\text { Sales in } \\
\text { Total TV } \\
\text { Sales }\end{array}$ \\
\hline 1998 & 5.794 .771 & 4.845 .796 & 0,84 & 6.331 .287 & 1.485 .491 & 0,23 \\
\hline 1999 & 6.941 .004 & 5.425 .734 & 0,78 & 6.852 .248 & 1.426 .514 & 0,21 \\
\hline 2000 & 8.788 .294 & 7.287 .323 & $\overline{0,83}$ & 8.730 .738 & 1.443 .415 & 0,17 \\
\hline 2001 & 8.025 .118 & 6.978 .610 & 0,87 & 8.009 .956 & 1.031 .346 & 0,13 \\
\hline 2002 & 12.462 .924 & 11.726 .289 & 0,94 & 12.368 .609 & 642.320 & 0,05 \\
\hline 2003 & 15.035 .590 & 14.836 .697 & 0,99 & 15.247 .245 & 410.548 & 0,03 \\
\hline 2004 & 20.345 .757 & 18.583 .429 & $\overline{0,91}$ & 19.954 .095 & 1.370 .666 & 0,07 \\
\hline 2005 & 20.790 .123 & 16.942 .529 & $\overline{0,81}$ & 19.125 .701 & 2.183 .172 & 0,11 \\
\hline 2006 & 17.930 .371 & 14.680 .350 & $\overline{0,82}$ & 16.614 .095 & 1.933 .745 & 0,12 \\
\hline 2007 & $12.591 .569^{\mathrm{a}}$ & $8.708 .505^{\mathrm{a}}$ & $0,69^{\mathrm{a}}$ & $11.900 .974^{\mathrm{a}}$ & $3.192 .469^{\mathrm{a}}$ & $0,27^{\mathrm{a}}$ \\
\hline \multicolumn{7}{|c|}{ * Calculated from Total Sales and Export figures and therefore does not include the sales of imported brands. } \\
\hline \multicolumn{7}{|c|}{${ }^{\mathrm{a}}$ Estimated figure. } \\
\hline \multicolumn{7}{|c|}{${ }^{\mathrm{b}}$ Source: TÜİK (2009). } \\
\hline \multicolumn{7}{|c|}{${ }^{\mathrm{c}}$ Sales figures for the years $2005-2007$ were unavailable for $51 \mathrm{~cm}$ and $55 \mathrm{~cm}$ television sets. } \\
\hline & & & & & & \\
\hline
\end{tabular}

It is hard to miss, however, the recent trend of decline in the year-on-year growth rate. As Europe constitutes the major export market of Turkish TVs, unfavorable economic developments in Europe as well as increasing competition in the European market seem to immediately result in repercussions for Turkish TV manufacturers. One of the reasons for the declining production may be the increasing consumer preference for LCD and plasma televisions in recent years. Another reason may be Turkish manufacturers' increasingly shifting their manufacturing operations abroad.

There are 326 firms operating in the industry of TV-radio receivers and audiovisual equipment, employing around 9,000 workers (Taylan and Sar1, 2008: 2). The main factories are located in Istanbul and Manisa (Taylan and Sari, 2008: 2). The leading manufacturers of television sets are ArçelikBeko (Beko Elektronik is now Grundig Elektronik, which merged entirely with Arçelik in late February of 2009), Vestel, and Profilo (Ministry of Industry and Trade, 2008).

\section{Television Exports}

In consumer electronics exports, color TV tops the list, accounting for $94.4 \%$ of all industry exports in 2007 (TÜİK 2008b), and accounting for over 90\% of all industry exports consistently since 1999 (Please see Table 3). In fact, since 1991 Turkey has enjoyed the position of net exporter of television sets. 
Since 1990 manufacturers of consumer electronics in Europe have lost market share due to the high costs of labor and material (Çakır, 2004: 12). Turkish manufacturers have used the advantage of low labor costs to enter the European market. The application of anti-dumping restrictions against Far Eastern companies in mid-1990s provided an additional advantage for Turkish companies, which have been insulated from the competition of their Far Eastern rivals (ibid.). In recent years, this advantage seems to be eroding as Far Eastern companies establish production centers in Europe in order to bypass anti-dumping regulations and customs barriers.

Table 3: Turkey's Television Exports, 1998-2007

\begin{tabular}{|l|c|c|c|c|}
\hline & TV Exports & TV Exports & $\begin{array}{c}\text { Total Consumer } \\
\text { Electronics } \\
\text { Exports }\end{array}$ & $\begin{array}{l}\text { Share of TV } \\
\text { exports in total } \\
\text { consumer } \\
\text { electronics }\end{array}$ \\
\cline { 2 - 5 } & (units) & (US dollars) & (US dollars) & \\
\hline 1998 & 4.845 .796 & 735.933 .788 & 837.555 .978 & 0,88 \\
\hline 1999 & 5.425 .734 & 674.894 .281 & 736.649 .395 & 0,92 \\
\hline 2000 & 7.287 .323 & 839.858 .926 & 890.169 .624 & 0,94 \\
\hline 2001 & 6.978 .610 & 864.986 .214 & 921.778 .005 & 0,94 \\
\hline 2002 & 11.726 .289 & 1.465 .640 .596 & 1.517 .668 .540 & 0,97 \\
\hline 2003 & 14.836 .697 & 1.820 .244 .134 & 1.908 .326 .713 & 0,95 \\
\hline 2004 & 18.583 .429 & 2.680 .456 .842 & 2.848 .708 .417 & 0,94 \\
\hline 2005 & 16.942 .529 & 2.933 .948 .995 & 3.130 .934 .434 & 0,94 \\
\hline 2006 & 14.680 .350 & 2.421 .853 .638 & 2.544 .743 .822 & 0,95 \\
\hline 2007 & $8.708 .505^{\mathrm{a}}$ & $2.025 .390 .007^{\mathrm{a}}$ & $2.144 .759 .796^{\mathrm{a}}$ & $0,94^{\mathrm{a}}$ \\
\hline${ }^{\mathrm{a}}$ Estimated figure. & & & \\
\hline * Source: TÜiK Turkish Statistics Institute, $2008 \mathrm{~b}$. & & \\
\hline
\end{tabular}

In fact, investments in recent years have been directed towards the former Eastern Bloc countries such as Hungary, Poland, and the Czech Republic as well as to countries where cheap and quality labor is readily available (Çakır, 2004: 13). Special privileges granted by some governments in Europe to attract foreign direct investment have exacerbated competition (ibid.).

Export numbers, as growth in production, also indicate a declining trend in recent years. One of the reasons may be the appreciating Turkish lira. Another may be Turkish manufacturers' increasingly shifting their manufacturing facilities abroad. Yet another reason may be the growing consumer shift away from cathode-ray tube televisions, which constitute the bulk of Turkish television manufacturing, towards LCD and plasma sets.

Examination of exported television sets by type seems to confirm this trend (Please see Table 4). While television sets with a screen size smaller than $42 \mathrm{~cm}$ had a share of $40 \%$ among total television export shipments for 1998, their share has fallen to $28 \%$ in 2006 and 2007. The share of the television set category with a screen size above 52 centimeters and below 72 centimeters, on the other hand, jumped from 1\% in 1999 to $46 \%$ in 2000, and in 2002 constituted $47 \%$ of total TV exports. In 2006 this category's share was $29 \%$ in total TV exports. Parallel with consumer trends, the above-72 cm screen CRT televisions have increased their share in TV exports consistently between 1998 and 2006, accounting for 4\% of total TV shipments abroad in 2006 and 5\% of total TV exports in value the same year. 
Table 4: Turkey's Television Exports by Television Type (units)

\begin{tabular}{|l|c|c|c|c|c|c|}
\hline \multicolumn{7}{|c|}{ TURKEY'S TELEVISION EXPORTS FOR 1998-2007 (Units) } \\
\hline & $\mathbf{1 9 9 8}$ & $\mathbf{1 9 9 9}$ & $\mathbf{2 0 0 0}$ & $\mathbf{2 0 0 1}$ & $\mathbf{2 0 0 2}$ & $\mathbf{2 0 0 3}$ \\
\hline $\begin{array}{l}\text { CRT TV }(\text { screen } \\
\text { diagonal<42 cm) }\end{array}$ & 1.952 .360 & 2.274 .311 & 2.732 .193 & 2.169 .596 & 3.586 .782 & 4.167 .003 \\
\hline $\begin{array}{l}\text { CRT TV }(42 \\
\text { cm<screen diagonal } \\
<52 \text { cm) }\end{array}$ & 946.468 & 1.125 .803 & 1.511 .677 & 1.253 .942 & 1.888 .235 & 1.973 .792 \\
\hline $\begin{array}{l}\text { CRT TV }(52 \\
\text { cm<screen diagonal } \\
<72 \text { cm) }\end{array}$ & 1.909 .079 & 1.921 .015 & 2.803 .359 & 3.213 .177 & 5.505 .734 & 6.167 .973 \\
\hline $\begin{array}{l}\text { CRT TV (screen } \\
\text { diagonal }>72 \text { cm) }\end{array}$ & 26.119 & 46.640 & 104.185 & 172.536 & 257.177 & 284.104 \\
\hline CRT TV Subtotal & $\mathbf{4 . 8 3 4 . 0 2 6}$ & $\mathbf{5 . 3 6 7 . 7 6 9}$ & $\mathbf{7 . 1 5 1 . 4 1 4}$ & $\mathbf{6 . 8 0 9 . 2 5 1}$ & $\mathbf{1 1 . 2 3 7 . 9 2 8}$ & $\mathbf{1 2 . 5 9 2 . 8 7 2}$ \\
\hline CRT TV Other & 1.301 & 12.755 & 59.720 & 94.723 & 175.490 & 468.267 \\
\hline CRT TV Total & $\mathbf{4 . 8 3 5 . 3 2 7}$ & $\mathbf{5 . 3 8 0 . 5 2 4}$ & $\mathbf{7 . 2 1 1 . 1 3 4}$ & $\mathbf{6 . 9 0 3 . 9 7 4}$ & $\mathbf{1 1 . 4 1 3 . 4 1 8}$ & $\mathbf{1 3 . 0 6 1 . 1 3 9}$ \\
\hline Combined TV & 4.731 & 1.851 & 1.760 & 9.894 & 238.841 & 709.321 \\
\hline $\begin{array}{l}\text { Video tuners with } \\
\text { screen and others }\end{array}$ & 392 & 2.662 & 2.740 & 35 & 4.231 & 887.807 \\
\hline Other TV & 5.346 & 40.697 & 71.689 & 64.707 & 69.799 & 178.430 \\
\hline TV TOTAL* & $\mathbf{4 . 8 4 5 . 7 9 6}$ & $\mathbf{5 . 4 2 5 . 7 3 4}$ & $\mathbf{7 . 2 8 7 . 3 2 3}$ & $\mathbf{6 . 9 7 8 . 6 1 0}$ & $\mathbf{1 1 . 7 2 6 . 2 8 9}$ & $\mathbf{1 4 . 8 3 6 . 6 9 7}$ \\
\hline $\begin{array}{l}\text { Total Consumer } \\
\text { Electronics }\end{array}$ & $\mathbf{4 . 9 1 6 . 9 5 5}$ & $\mathbf{5 . 5 1 4 . 0 7 0}$ & $\mathbf{7 . 3 9 2 . 6 7 5}$ & $\mathbf{7 . 1 4 8 . 1 0 1}$ & $\mathbf{3 5 . 0 9 6 . 9 3 3}$ & $\mathbf{7 1 . 8 2 9 . 0 0 1}$ \\
\hline
\end{tabular}

\begin{tabular}{|c|c|c|c|c|c|}
\hline & 2004 & 2005 & 2006 & & $2007 * *$ \\
\hline $\begin{array}{l}\text { CRT TV }(\text { screen } \\
\text { diagonal }<42 \mathrm{~cm})\end{array}$ & 4.880 .936 & 4.212 .850 & 4.124 .177 & $\begin{array}{l}\text { CRT TV (screen diago- } \\
\text { nal<42 cm) }\end{array}$ & 2.473 .404 \\
\hline $\begin{array}{l}\text { CRT TV }(42 \\
\mathrm{cm}<\text { screen diagonal } \\
<52 \mathrm{~cm})\end{array}$ & 2.259 .137 & 1.676 .881 & 1.749 .455 & $\begin{array}{l}\text { CRT TV }(42 \mathrm{~cm}<\text { screen } \\
\text { diagonal }<52 \mathrm{~cm})\end{array}$ & \\
\hline $\begin{array}{l}\text { CRT TV }(52 \\
\mathrm{cm}<\text { screen diagonal } \\
<72 \mathrm{~cm})\end{array}$ & 6.439 .098 & 5.322 .690 & 4.303 .697 & $\begin{array}{l}\text { CRT TV }(52 \mathrm{~cm}<\text { screen } \\
\text { diagonal }<72 \mathrm{~cm})\end{array}$ & 1.125 .762 \\
\hline $\begin{array}{l}\text { CRT TV }(\text { screen } \\
\text { diagonal }>72 \mathrm{~cm})\end{array}$ & 483.329 & 528.360 & 649.882 & $\begin{array}{l}\text { CRT TV (screen diagonal } \\
>72 \mathrm{~cm})\end{array}$ & 48.448 \\
\hline CRT TV Subtotal & 14.062 .500 & 11.740 .781 & 10.827 .211 & CRT TV Subtotal & 3.647 .614 \\
\hline CRT TV Other & 1.095 .520 & 822.390 & 486.979 & CRT TV Other**** & \\
\hline CRT TV Total & 15.158 .020 & 12.563 .171 & 11.314 .190 & CRT TV Total & 3.647 .614 \\
\hline Combined TV & 2.060 .656 & 1.238 .159 & 1.007 .722 & Plasma TV*** & \\
\hline $\begin{array}{l}\text { Video tuners with } \\
\text { screen and others }\end{array}$ & 791.076 & 2.347 .339 & 1.899 .632 & LCD TV & 4.331 .682 \\
\hline Other TV & 573.677 & 793.860 & 458.806 & Other TV & 729.209 \\
\hline TV TOTAL* & 18.583 .429 & 16.942 .529 & 14.680 .350 & TV TOTAL* & 8.708 .505 \\
\hline $\begin{array}{l}\text { Total Consumer } \\
\text { Electronics }\end{array}$ & 70.388 .814 & 65.398 .984 & 21.204 .977 & Total Consumer Electronics & 45.103 .024 \\
\hline \multicolumn{6}{|c|}{$\begin{array}{l}\text { * The customs tariff position number " } 85-28 \text { " includes besides television sets, teleprojectors, } \\
\text { color video projectors, and satellite television receivers. Since the latter constitutes only a small } \\
\text { segment within the category, they were included in "Other TV" and hence "TV TOTAL". }\end{array}$} \\
\hline \multicolumn{6}{|c|}{$* *$ The 2007 figures are provisional. } \\
\hline \multicolumn{6}{|c|}{$\begin{array}{l}* * * \text { According to the legislation pertaining to data confidentiality, certain figures were not re- } \\
\text { leased. }\end{array}$} \\
\hline
\end{tabular}


Table 5: Turkey's Television Exports by Television Type (US dollars) TURKEY'S TELEVISION EXPORTS FOR 1998-2007 (US Dollars)

\begin{tabular}{|c|c|c|c|c|c|c|}
\hline & 1998 & 1999 & 2000 & 2001 & 2002 & 2003 \\
\hline $\begin{array}{l}\text { CRT TV (screen } \\
\text { diagonal }<42 \mathrm{~cm} \text { ) }\end{array}$ & 204.478 .796 & 197.760 .623 & 224.046 .150 & 172.871 .519 & 259.705 .693 & 307.378 .741 \\
\hline $\begin{array}{l}\text { CRT TV ( } 42 \\
\mathrm{~cm}<\mathrm{screen} \text { diagonal } \\
<52 \mathrm{~cm})\end{array}$ & 129.532 .497 & 127.159 .027 & 151.183 .617 & 124.153 .239 & 173.710 .558 & 202.483 .561 \\
\hline $\begin{array}{l}\text { CRT TV (52 } \\
\mathrm{cm}<\mathrm{screen} \text { diagonal } \\
<72 \mathrm{~cm})\end{array}$ & 387.768 .643 & 324.979 .285 & 413.146 .267 & 480.970 .619 & 826.486 .874 & 898.833 .102 \\
\hline $\begin{array}{l}\text { CRT TV (screen } \\
\text { diagonal > } 72 \mathrm{~cm} \text { ) }\end{array}$ & 13.018 .676 & 16.103 .822 & 27.250 .205 & 46.522 .459 & 74.721 .655 & 88.109 .661 \\
\hline CRT TV Subtotal & 734.798.612 & 666.002 .757 & 815.626.239 & 824.517 .836 & 1.334.624.780 & 1.496.805.065 \\
\hline CRT TV Other & 329.660 & 4.727 .409 & 20.550 .096 & 35.657 .764 & 69.629 .489 & 142.366 .616 \\
\hline CRT TV Total & 735.128 .272 & 670.730 .166 & 836.176 .335 & 860.175 .600 & 1.404 .254 .269 & 1.639 .171 .681 \\
\hline Combined TV & 174.580 & 183.515 & 266.322 & 2.067 .893 & 52.686 .504 & 103.493 .453 \\
\hline $\begin{array}{l}\text { Video tuners with } \\
\text { screen and others }\end{array}$ & 189.154 & 299.668 & 320.087 & 51.751 & 1.365 .788 & 22.052 .121 \\
\hline Other TV & 441.782 & 3.680 .932 & 3.096 .182 & 2.690 .970 & 7.334 .035 & 55.526 .879 \\
\hline TV TOTAL* & 735.933.788 & 674.894.281 & 839.858 .926 & 864.986 .214 & 1.465 .640 .596 & 1.820 .244 .134 \\
\hline $\begin{array}{l}\text { Total Consumer } \\
\text { Electronics }\end{array}$ & 837.555.978 & 736.649.395 & 890.169 .624 & 921.778.005 & 1.517.668.540 & 1.908.326.713 \\
\hline
\end{tabular}

\begin{tabular}{|c|c|c|c|c|c|}
\hline & 2004 & 2005 & 2006 & & $2007^{* * *}$ \\
\hline $\begin{array}{l}\text { CRT TV (screen } \\
\text { diagonal }<42 \mathrm{~cm} \text { ) }\end{array}$ & 341.919 .266 & 255.832 .067 & 244.945 .696 & CRT TV (screen diagonal $<42 \mathrm{~cm}$ ) & 153.098 .427 \\
\hline $\begin{array}{l}\text { CRT TV ( } 42 \\
\mathrm{~cm}<\mathrm{screen} \text { diagonal } \\
<52 \mathrm{~cm})\end{array}$ & 237.410 .756 & 137.217.447 & 140.764 .025 & CRT TV $(42 \mathrm{~cm}<$ screen diagonal $<52 \mathrm{~cm})$ & \\
\hline $\begin{array}{l}\text { CRT TV (52 } \\
\mathrm{cm}<\text { screen diagonal } \\
<72 \mathrm{~cm} \text { ) }\end{array}$ & 932.144 .428 & 603.153 .852 & 474.299 .719 & CRT TV $(52 \mathrm{~cm}<$ screen diagonal $<72 \mathrm{~cm})$ & 158.795 .809 \\
\hline $\begin{array}{l}\text { CRT TV (screen } \\
\text { diagonal }>72 \mathrm{~cm} \text { ) }\end{array}$ & 128.984 .957 & 110.263 .774 & 119.259 .210 & CRT TV (screen diagonal $>72 \mathrm{~cm}$ ) & 9.799 .965 \\
\hline CRT TV Subtotal & 1.640 .459 .407 & 1.106 .467 .140 & 979.268.650 & CRT TV Subtotal & 321.694.201 \\
\hline CRT TV Other & 290.330 .704 & 180.491 .360 & 103.952.241 & CRT TV Other*** & \\
\hline CRT TV Total & 1.930 .790 .111 & 1.286.958.500 & 1.083.220.891 & CRT TV Total & 321.694 .201 \\
\hline Combined TV & 182.193 .457 & 141.802 .774 & 110.611 .827 & Plasma TV*** & \\
\hline $\begin{array}{l}\text { Video tuners with } \\
\text { screen and others }\end{array}$ & 501.406 .649 & 1.336 .441 .842 & 1.206.770.624 & LCD TV & 1.667.533.396 \\
\hline Other TV & 66.066 .625 & 168.745 .879 & 21.250 .296 & Other TV & 36.162 .410 \\
\hline TV TOTAL* & 2.680 .456 .842 & 2.933.948.995 & 2.421.853.638 & TV TOTAL* & 2.025.390.007 \\
\hline $\begin{array}{l}\text { Total Consumer } \\
\text { Electronics }\end{array}$ & 2.848.708.417 & 3.130.934.434 & 2.544.743.822 & Total Consumer Electronics & 2.144.759.796 \\
\hline
\end{tabular}

* The customs tariff position number "85-28" includes besides television sets, teleprojectors, color video projectors, and satellite television receivers. Since the latter constitutes only a small segment within the category, they were included in "Other TV" and hence "TV TOTAL".

** The 2007 figures are provisional.

*** According to the legislation pertaining to data confidentiality, certain figures were not released. Source: TÜIK, 2008 b.

Declining TV prices is a well-known phenomenon around the world, and this trend can easily be seen in the mean unit prices of Turkish TV exports and imports across the years. 
Table 6: Mean Unit Prices of TV Export Items, 1998-2003

\begin{tabular}{|c|c|c|c|c|c|c|c|c|c|}
\hline $\begin{array}{l}\text { EXPORT } \\
\text { ITEM }\end{array}$ & $\begin{array}{r}\text { Amount } \\
\text { (Units) }\end{array}$ & Value (USD) & $\begin{array}{r}\text { Mean Unit } \\
\text { Price (USD) }\end{array}$ & $\begin{array}{r}\text { Amount } \\
\text { (Units) }\end{array}$ & Value (USD) & $\begin{array}{r}\text { Mean } \\
\text { Unit Price } \\
\text { (USD) }\end{array}$ & Amount (Units) & Value (USD) & $\begin{array}{r}\text { Mean Unit } \\
\text { Price (USD) }\end{array}$ \\
\hline & 1998 & 1998 & 1998 & 1999 & 1999 & 1999 & 2000 & 2000 & 2000 \\
\hline $\begin{array}{l}\text { CRT TV } \\
\text { (screen } \\
\text { diagonal<42 } \\
\mathrm{cm} \text { ) }\end{array}$ & 1.952 .360 & 204.478 .796 & 104,73 & 2.274 .311 & 197.760 .623 & 86,95 & 2.732 .193 & 224.046 .150 & 82,00 \\
\hline $\begin{array}{l}\text { CRT TV } \\
(42 \\
\mathrm{cm}<\text { screen } \\
\text { diagonal } \\
<52 \mathrm{~cm})\end{array}$ & 946.468 & 129.532 .497 & 136,86 & 1.125 .803 & 127.159.027 & 112,95 & 1.511 .677 & 151.183 .617 & 100,01 \\
\hline $\begin{array}{l}\text { CRT TV } \\
(52 \\
\mathrm{cm}<\text { screen } \\
\text { diagonal } \\
<72 \mathrm{~cm})\end{array}$ & 1.909 .079 & 387.768 .643 & 203,12 & 1.921 .015 & 324.979 .285 & 169,17 & 2.803 .359 & 413.146 .267 & 147,38 \\
\hline $\begin{array}{l}\text { CRT TV } \\
\text { (screen } \\
\text { diagonal > } \\
72 \mathrm{~cm} \text { ) }\end{array}$ & 26.119 & 13.018 .676 & 498,44 & 46.640 & 16.103 .822 & 345,28 & 104.185 & 27.250 .205 & 261,56 \\
\hline $\begin{array}{l}\text { CRT TV } \\
\text { Subtotal }\end{array}$ & 4.834.026 & 734.798.612 & 152,01 & 5.367 .769 & 666.002 .757 & 124,07 & 7.151 .414 & 815.626.239 & 114,05 \\
\hline $\begin{array}{l}\text { CRT TV } \\
\text { Other }\end{array}$ & 1.301 & 329.660 & 253,39 & 12.755 & 4.727 .409 & 370,63 & 59.720 & 20.550 .096 & 344,11 \\
\hline $\begin{array}{l}\text { CRT TV } \\
\text { Total }\end{array}$ & 4.835.327 & 735.128 .272 & 152,03 & 5.380 .524 & 670.730 .166 & 124,66 & 7.211.134 & 836.176 .335 & 115,96 \\
\hline $\begin{array}{l}\text { Combined } \\
\text { TV }\end{array}$ & 4.731 & 174.580 & 36,90 & 1.851 & 183.515 & 99,14 & 1.760 & 266.322 & 151,32 \\
\hline $\begin{array}{l}\text { Video } \\
\text { tuners with } \\
\text { screen, etc. }\end{array}$ & 392 & 189.154 & 482,54 & 2.662 & 299.668 & 112,57 & 2.740 & 320.087 & 116,82 \\
\hline Other TV & 5.346 & 441.782 & 82,64 & 40.697 & 3.680 .932 & 90,45 & 71.689 & 3.096 .182 & 43,19 \\
\hline \begin{tabular}{|l} 
TV \\
TOTAL*
\end{tabular} & 4.845.796 & 735.933.788 & 151,87 & 5.425 .734 & 674.894.281 & 124,39 & 7.287.323 & 839.858.926 & 115,25 \\
\hline $\begin{array}{l}\text { Total } \\
\text { Consumer } \\
\text { Electronics }\end{array}$ & 4.916 .955 & 837.555.978 & & 5.514 .070 & 736.649.395 & & 7.392.675 & 890.169 .624 & \\
\hline
\end{tabular}

\begin{tabular}{|c|c|c|c|c|c|c|c|c|c|}
\hline $\begin{array}{l}\text { EXPORT } \\
\text { ITEM }\end{array}$ & $\begin{array}{r}\text { Amount } \\
\text { (Units) }\end{array}$ & Value (USD) & $\begin{array}{l}\text { Mean Unit } \\
\text { Price (USD) }\end{array}$ & $\begin{array}{r}\text { Amount } \\
\text { (Units) }\end{array}$ & Value (USD) & $\begin{array}{l}\text { Mean Unit } \\
\text { Price(USD) }\end{array}$ & $\begin{array}{r}\text { Amount } \\
\text { (Units) }\end{array}$ & Value (USD) & $\begin{array}{l}\text { Mean Unit } \\
\text { Price (USD) }\end{array}$ \\
\hline & 2001 & 2001 & 2001 & 2002 & 2002 & 2002 & 2003 & 2003 & 2003 \\
\hline $\begin{array}{l}\text { CRT TV } \\
\text { (screen } \\
\text { diagonal<42 } \\
\mathrm{cm} \text { ) }\end{array}$ & 2.169 .596 & 172.871 .519 & 79,68 & 3.586 .782 & 259.705 .693 & 72,41 & 4.167 .003 & 307.378 .741 & 73,76 \\
\hline $\begin{array}{l}\text { CRT TV (42 } \\
\text { cm<screen } \\
\text { diagonal }<52 \\
\mathrm{~cm})\end{array}$ & 1.253 .942 & 124.153 .239 & 99,01 & 1.888 .235 & 173.710 .558 & 92,00 & 1.973 .792 & 202.483 .561 & 102,59 \\
\hline $\begin{array}{l}\text { CRT TV }(52 \\
\mathrm{cm}<\text { screen } \\
\text { diagonal }<72 \\
\mathrm{~cm})\end{array}$ & 3.213 .177 & 480.970 .619 & 149,69 & 5.505 .734 & 826.486 .874 & 150,11 & 6.167 .973 & 898.833 .102 & 145,73 \\
\hline $\begin{array}{l}\text { CRT TV } \\
\text { (screen } \\
\text { diagonal >72 } \\
\text { cm) }\end{array}$ & 172.536 & 46.522 .459 & 269,64 & 257.177 & 74.721 .655 & 290,55 & 284.104 & 88.109 .661 & 310,13 \\
\hline $\begin{array}{l}\text { CRT TV } \\
\text { Subtotal }\end{array}$ & 6.809 .251 & 824.517.836 & 121,09 & 11.237.928 & 1.334 .624 .780 & 118,76 & 12.592 .872 & 1.496 .805 .065 & 118,86 \\
\hline $\begin{array}{l}\text { CRT TV } \\
\text { Other }\end{array}$ & 94.723 & 35.657 .764 & 376,44 & 175.490 & 69.629 .489 & 396,77 & 468.267 & 142.366 .616 & 304,03 \\
\hline $\begin{array}{l}\text { CRT TV } \\
\text { TOTAL }\end{array}$ & 6.903 .974 & 860.175 .600 & 124,59 & 11.413.418 & 1.404 .254 .269 & 123,04 & 13.061.139 & 1.639 .171 .681 & 125,50 \\
\hline $\begin{array}{l}\text { COMBINED } \\
\text { TV }\end{array}$ & 9.894 & 2.067 .893 & 209,00 & 238.841 & 52.686 .504 & 220,59 & 709.321 & 103.493 .453 & 145,90 \\
\hline $\begin{array}{l}\text { VIDEO } \\
\text { TUNERS } \\
\text { WITH } \\
\text { SCREEN and } \\
\text { others }\end{array}$ & 35 & 51.751 & $1.478,60$ & 4.231 & 1.365 .788 & 322,81 & 887.807 & 22.052 .121 & 24,84 \\
\hline Other TV & 64.707 & 2.690 .970 & 41,59 & 69.799 & 7.334 .035 & 105,07 & 178.430 & 55.526 .879 & 311,20 \\
\hline TV Total* & 6.978.610 & 864.986 .214 & 123,95 & 11.726 .289 & 1.465 .640 .596 & 124,99 & 14.836.697 & 1.820 .244 .134 & 122,69 \\
\hline \begin{tabular}{|l} 
Total \\
Consumer \\
Electronics
\end{tabular} & 7.148.101 & 921.778.005 & & 35.096 .933 & 1.517 .668 .540 & & 71.829.001 & 1.908 .326 .713 & \\
\hline
\end{tabular}


* The customs tariff position number "85-28" includes besides television sets, teleprojectors, color video projectors, and satellite television receivers. Since the latter constitutes only a small segment within the category, they were included in "Other TV" and hence "TV TOTAL".

** The 2007 figures are provisional.

*** According to the legislation pertaining to data confidentiality, certain figures were not released.

Source: TÜIKK, 2008b.

Table 7: Mean Unit Prices of TV Export Items, 2004-2007

\begin{tabular}{|c|c|c|c|c|c|c|c|c|c|}
\hline $\begin{array}{l}\text { EXPORT } \\
\text { ITEM }\end{array}$ & $\begin{array}{r}\text { Amount } \\
\text { (Units) }\end{array}$ & Value (USD) & $\begin{array}{r}\text { Mean } \\
\text { Unit } \\
\text { Price } \\
\text { (USD) } \\
\end{array}$ & $\begin{array}{r}\text { Amount } \\
\text { (Units) }\end{array}$ & Value (USD) & $\begin{array}{r}\text { Mean Unit } \\
\text { Price (USD) }\end{array}$ & $\begin{array}{r}\text { Amount } \\
\text { (Units) }\end{array}$ & Value (USD) & $\begin{array}{r}\text { Mean } \\
\text { Unit Price } \\
\text { (USD) }\end{array}$ \\
\hline & 2004 & 2004 & 2004 & 2005 & 2005 & 2005 & 2006 & 2006 & 2006 \\
\hline $\begin{array}{l}\text { CRT TV } \\
\text { (screen } \\
\text { diagonal<42 } \\
\mathrm{cm} \text { ) } \\
\end{array}$ & 4.880 .936 & 341.919 .266 & 70,05 & 4.212 .850 & 255.832 .067 & 60,73 & 4.124 .177 & 244.945 .696 & 59,39 \\
\hline $\begin{array}{l}\text { CRT TV }(42 \\
\mathrm{cm}<\text { screen } \\
\text { diagonal } \\
<52 \mathrm{~cm}) \\
\end{array}$ & 2.259 .137 & 237.410 .756 & 105,09 & 1.676 .881 & 137.217 .447 & 81,83 & 1.749 .455 & 140.764 .025 & 80,46 \\
\hline $\begin{array}{l}\text { CRT TV }(52 \\
\mathrm{cm}<\text { screen } \\
\text { diagonal } \\
<72 \mathrm{~cm}) \\
\end{array}$ & 6.439 .098 & 932.144 .428 & 144,76 & 5.322 .690 & 603.153 .852 & 113,32 & 4.303 .697 & 4744.299 .719 & 110,21 \\
\hline $\begin{array}{l}\text { CRT TV } \\
\text { (screen } \\
\text { diagonal } \\
>72 \mathrm{~cm} \text { ) } \\
\end{array}$ & 483.329 & 128.984 .957 & 266,87 & 528.360 & 110.263 .774 & 208,69 & 649.882 & 119.259 .210 & 183,51 \\
\hline $\begin{array}{l}\text { CRT TV } \\
\text { Subtotal }\end{array}$ & 14.062 .500 & 1.640 .459 .407 & 116,65 & 11.740 .781 & 1.106 .467 .140 & 94,24 & 10.827 .211 & 979.268 .650 & 90,45 \\
\hline $\begin{array}{l}\text { CRT TV } \\
\text { Other }\end{array}$ & 1.095 .520 & 290.330 .704 & 265,02 & 822.390 & 180.491 .360 & 219,47 & 486.979 & 103.952 .241 & 213,46 \\
\hline $\begin{array}{l}\text { CRT TV } \\
\text { Total }\end{array}$ & 15.158 .020 & 1.930 .790 .111 & 127,38 & 12.563 .171 & 1.286 .958 .500 & 102,44 & 11.314 .190 & 1.083 .220 .891 & 95,74 \\
\hline $\begin{array}{l}\text { Combined } \\
\text { TV }\end{array}$ & 2.060 .656 & 182.193 .457 & 88,42 & 1.238 .159 & 141.802 .774 & 114,53 & 1.007 .722 & 110.611 .827 & 109,76 \\
\hline $\begin{array}{l}\text { Video tuners } \\
\text { with screen, } \\
\text { etc. }\end{array}$ & 791.076 & 501.406 .649 & 633,83 & 2.347 .339 & 1.336 .441 .842 & 569,34 & 1.899 .632 & 1.206 .770 .624 & 635,27 \\
\hline Other TV & 573.677 & 66.066 .625 & 115,16 & 793.860 & 168.745 .879 & 212,56 & 458.806 & 21.250 .296 & 46,32 \\
\hline $\begin{array}{l}\text { TV } \\
\text { TOTAL** }\end{array}$ & 18.583 .429 & 2.680 .456 .842 & 144,24 & 16.942 .529 & 2.933 .948 .995 & 173,17 & 14.680 .350 & 2.421 .853 .638 & 164,97 \\
\hline $\begin{array}{l}\text { Total } \\
\text { Consumer } \\
\text { Electronics } \\
\end{array}$ & 70.388 .814 & 2.848 .708 .417 & & 65.398 .984 & 3.130 .934 .434 & & 21.204 .977 & 2.544 .743 .822 & \\
\hline
\end{tabular}

\begin{tabular}{|c|c|c|c|}
\hline Export Item & Amount (Units) & Value (USD) & Mean Unit Price (USD) \\
\hline & $2007 * *$ & $2007^{* *}$ & $2007^{* * *}$ \\
\hline CRT TV (screen diagonal $<42 \mathrm{~cm}$ ) & 2.473 .404 & 153.098 .427 & 61,90 \\
\hline \multicolumn{4}{|l|}{ CRT TV $(42 \mathrm{~cm}<$ screen diagonal $<52 \mathrm{~cm})$} \\
\hline CRT TV $(52 \mathrm{~cm}<$ screen diagonal $<72 \mathrm{~cm})$ & 1.125 .762 & 158.795 .809 & 141,06 \\
\hline CRT TV (screen diagonal $>72 \mathrm{~cm}$ ) & 48.448 & 9.799 .965 & 202,28 \\
\hline CRT TV Subtotal & 3.647 .614 & 321.694.201 & 88,19 \\
\hline \multicolumn{4}{|l|}{ CRT TV Other*** } \\
\hline CRT TV Total & 3.647.614 & 321.694 .201 & 88,19 \\
\hline \multicolumn{4}{|l|}{ Plasma TV*** } \\
\hline LCD TV & 4.331 .682 & 1.667 .533 .396 & 384,96 \\
\hline Other TV & 729.209 & 36.162 .410 & 49,59 \\
\hline TV TOTAL* & 8.708 .505 & 2.025.390.007 & 232,58 \\
\hline Total Consumer Electronics & 45.103.024,00 & 2.144 .759 .796 & \\
\hline \multicolumn{4}{|c|}{$\begin{array}{l}\text { * The customs tariff position number " } 85-28 \text { " includes besides television sets, teleprojectors, color video projectors, and satellite television receivers. Since the } \\
\text { latter constitutes only a small segment within the category, they were included in "Other TV" and hence "TV TOTAL". }\end{array}$} \\
\hline \multicolumn{4}{|l|}{ ** The 2007 figures are provisional. } \\
\hline \multicolumn{4}{|c|}{$* * *$ According to the legislation pertaining to data confidentiality, certain figures were not released. } \\
\hline Source: TUIK, 2008b. & & & \\
\hline
\end{tabular}

Color televisions made in Turkey have a good reputation among European consumers with respect to price and quality. Turkish manufacturers follow European and international standards and norms, and since 2002, production carries the "CE" mark (Taylan and Sarı, 2008: 3). 
The European Union is Turkey's biggest trading partner; in 2006, Turkey received 4.3\% of all EU exports, and 3.1\% of all EU imports that year were accounted for by Turkey (Eurostat, 2007: 57). In 2005, Turkey's global exports were valued at 58.8 billion Euros, $56 \%$ of which were destined to the EU-27; and that same year a share of $45 \%$ of imports to Turkey was accounted for by the EU-27.

Of total television exports, $91 \%$ are headed to ten member states of the European Union (DTM, 2006: 6). For television sets, the UK has been the biggest export market for the past two years, followed by Germany, Spain, France, the Netherlands, and Italy. Of total television exports for 2007, for instance, the UK accounted for 32\%, Germany for $20 \%$, Spain for $10 \%$, France for $9 \%$, the Netherlands for 5\%, and Italy for 4\% (DTM, 2006: 8).

Today Turkish producers command 25\% of the European color TV market (Önkol, 2008), and Turkey appears to be the television factory of Europe. The second biggest market for color TVs after the European Union is the Russian Federation and the Turkic republics (Çakır, 2004).

\section{Television Imports}

Electronic components production in Turkey is limited, and especially some of the components used in the manufacturing of television sets are largely imported (Çakır, 2004: 16). It is sometimes more cost-effective for Turkish manufacturers to import finished goods, especially from China, South Korea, and Hong Kong (ibid.). Turkey's television imports over the past ten years, therefore, follow a generally increasing trend, with occasional dips such as in the recession year of 2001 (Please see Table 8).

Between 2005 and 2006, for instance, TV imports rose by $22 \%$ in value, from US\$221,869,314 to US\$270,773,182. Television sets is an area, however, where Turkey has a favorable trade balance. Between 2002 and 2005, the export/import ratio has exceeded 1,300\% (reaching its peak of 1,612\% in 2002).

Table 8: Turkey's Television Exports and Imports, 1998-2007

\begin{tabular}{|l|r|r|r|}
\hline YEAR & EXPORTS & IMPORTS & EXPORT/IMPORT RATIO \\
\hline & (US Dollars) & (US Dollars) & $\%$ \\
\hline 1998 & 735.933 .788 & 93.254 .438 & 789,17 \\
\hline 1999 & 674.894 .281 & 113.123 .599 & 596,60 \\
\hline 2000 & 839.858 .926 & 178.476 .868 & 470,57 \\
\hline 2001 & 864.986 .214 & 97.090 .684 & 890,91 \\
\hline 2002 & 1.465 .640 .596 & 90.920 .719 & $1.612,00$ \\
\hline 2003 & 1.820 .244 .134 & 119.425 .348 & $1.524,17$ \\
\hline 2004 & 2.680 .456 .842 & 193.849 .072 & $1.382,75$ \\
\hline 2005 & 2.933 .948 .995 & 221.869 .314 & $1.322,38$ \\
\hline 2006 & 2.421 .853 .638 & 270.773 .182 & 894,42 \\
\hline 2007 & $2.025 .390 .007^{\mathrm{a}}$ & $760.191 .838^{\mathrm{a}}$ & $266,43^{\mathrm{a}}$ \\
\hline
\end{tabular}

${ }^{\mathrm{a}}$ The figures for 2007 are provisional, and according to the legislation pertaining to data confidentiality, data for some of the categories have not been released.

Source: TÜIK (Turkish Statistics Institute), 2008b. 
The consumer shift away from cathode-ray televisions towards LCD and plasma televisions can be clearly seen from the import figures of recent years (Please see Table 9). While the share of CRT televisions in total television imports was $41 \%$ in 1998, by 2006 that figure has fallen to $9 \%$ in unit terms. In dollar terms, that corresponds to a decline from $69 \%$ to $6 \%$.

Table 9: Turkey's Television Imports, 1998-2007 (Units)

\begin{tabular}{|c|c|c|c|c|c|c|}
\hline & 1998 & 1999 & 2000 & 2001 & 2002 & 2003 \\
\hline $\begin{array}{l}\text { CRT TV (screen diagonal }<42 \\
\mathrm{~cm} \text { ) }\end{array}$ & 118.008 & 230.252 & 193.538 & 64.252 & 118.496 & 228.850 \\
\hline $\begin{array}{l}\text { CRT TV }(42 \mathrm{~cm}<\text { screen } \\
\text { diagonal }<52 \mathrm{~cm})\end{array}$ & 83.938 & 67.039 & 68.029 & 23.928 & 16.831 & 10.591 \\
\hline $\begin{array}{l}\text { CRT TV }(52 \mathrm{~cm}<\text { screen } \\
\text { diagonal }<72 \mathrm{~cm})\end{array}$ & 97.719 & 137.014 & 186.605 & 50.948 & 127.583 & 112.361 \\
\hline $\begin{array}{l}\text { CRT TV (screen diagonal > } 72 \\
\mathrm{~cm} \text { ) }\end{array}$ & 9.953 & 10.501 & 38.514 & 25.696 & 45.675 & 57.180 \\
\hline CRT TV Subtotal & 309.618 & 444.806 & 486.686 & 164.824 & 308.585 & 408.982 \\
\hline CRT TV Other & 2.894 & 2.036 & 8.545 & 3.274 & 5.015 & 7.131 \\
\hline CRT TV Total & 312.512 & 446.842 & 495.231 & 168.098 & 313.600 & 416.113 \\
\hline Combined TV & 2.757 & 2.152 & 5.239 & 710 & 4.585 & 5.160 \\
\hline $\begin{array}{l}\text { Video tuners with screen and } \\
\text { others }\end{array}$ & 1.500 & 603 & 3.069 & 5.203 & 4.613 & 9.297 \\
\hline Other TV & 440.270 & 607.971 & 873.243 & 486.149 & 380.596 & 779.093 \\
\hline TV TOTAL* & 757.039 & 1.057 .568 & 1.376 .782 & 660.160 & 703.394 & 1.209 .663 \\
\hline Total Consumer Electronics & 8.253 .458 & 8.347 .346 & 10.582 .498 & 4.793.957 & 88.696.142 & 89.175.735 \\
\hline
\end{tabular}

\begin{tabular}{|c|c|c|c|c|c|}
\hline & 2004 & 2005 & 2006 & & $2007 * *$ \\
\hline $\begin{array}{l}\text { CRT TV (screen diagonal }<42 \\
\mathrm{~cm} \text { ) }\end{array}$ & 93.205 & 22.840 & 4.185 & CRT TV (screen diagonal $<42 \mathrm{~cm}$ ) & 8.239 \\
\hline $\begin{array}{l}\text { CRT TV }(42 \mathrm{~cm}<\text { screen diago- } \\
\text { nal }<52 \mathrm{~cm})\end{array}$ & 6.988 & 4.060 & 1.930 & $\begin{array}{l}\text { CRT TV }(42 \mathrm{~cm}<\text { screen diagonal }<52 \\
\mathrm{cm})\end{array}$ & 98 \\
\hline $\begin{array}{l}\text { CRT TV }(52 \mathrm{~cm}<\text { screen diago- } \\
\text { nal }<72 \mathrm{~cm})\end{array}$ & 70.812 & 65.226 & 29.546 & $\begin{array}{l}\text { CRT TV }(52 \mathrm{~cm}<\text { screen diagonal }<72 \\
\mathrm{cm})\end{array}$ & 17.321 \\
\hline $\begin{array}{l}\text { CRT TV (screen diagonal > } 72 \\
\mathrm{~cm} \text { ) }\end{array}$ & 86.205 & 56.574 & 21.867 & CRT TV (screen diagonal $>72 \mathrm{~cm}$ ) & 6.984 \\
\hline CRT TV Subtotal & 257.210 & 148.700 & $\mathbf{5 7 . 5 2 8}$ & CRT TV Subtotal & 32.642 \\
\hline CRT TV Other & 22.981 & 17.442 & 6.926 & CRT TV Other*** & 96 \\
\hline CRT TV Total & 280.191 & 166.142 & 64.454 & CRT TV Total & 32.738 \\
\hline Combined TV & 4.068 & 3.912 & 3.381 & Plasma TV**** & 49.577 \\
\hline $\begin{array}{l}\text { Video tuners with screen and } \\
\text { others }\end{array}$ & 24.051 & 67.968 & 140.890 & LCD TV & 266.647 \\
\hline Other TV & 2.035 .953 & 1.368 .152 & 476.109 & Other TV & 3.278 .167 \\
\hline TV TOTAL* & 2.344 .263 & 1.606 .174 & 684.834 & TV TOTAL* & 3.627.129 \\
\hline Total Consumer Electronics & 164.265 .132 & 119.422 .618 & 85.504.889 & Total Consumer Electronics & 601.478 .514 \\
\hline
\end{tabular}

* The customs tariff position number " $85-28$ " includes besides television sets, teleprojectors, color video projectors, and satellite television receivers. Since the latter constitutes only a small segment within the category, they were included in "Other TV" and hence "TV TOTAL".

** The 2007 figures are provisional.

*** According to the legislation pertaining to data confidentiality, certain figures were not released. Source: TÜIK, 2008 b. 
In television set imports, as in exports, European Union countries top the list. They are followed by Japan and other countries in the Far East.

Table 10: Turkey's Television Imports, 1998-2007 (US Dollars)

\begin{tabular}{|l|r|r|r|r|r|r|}
\hline & $\mathbf{1 9 9 8}$ & $\mathbf{1 9 9 9}$ & $\mathbf{2 0 0 0}$ & $\mathbf{2 0 0 1}$ & $\mathbf{2 0 0 2}$ & $\mathbf{2 0 0 3}$ \\
\hline $\begin{array}{l}\text { CRT TV }(\text { screen diago- } \\
\text { nal<42 cm) }\end{array}$ & 14.930 .651 & 16.384 .207 & 13.983 .065 & 4.765 .841 & 5.170 .565 & 8.844 .754 \\
\hline $\begin{array}{l}\text { CRT TV }(42 \mathrm{~cm} \text { <screen } \\
\text { diagonal } 55 \mathrm{~cm} \text { ) }\end{array}$ & 15.933 .906 & 10.216 .121 & 7.910 .909 & 2.525 .023 & 1.513 .993 & 1.076 .977 \\
\hline $\begin{array}{l}\text { CRT TV }(52 \mathrm{~cm} \text { <screen } \\
\text { diagonal }<72 \mathrm{~cm} \text { ) }\end{array}$ & 26.830 .377 & 31.042 .533 & 33.744 .650 & 8.951 .770 & 18.866 .235 & 12.430 .314 \\
\hline $\begin{array}{l}\text { CRT TV }(\text { screen diago- } \\
\text { nal }>72 \text { cm) }\end{array}$ & 4.913 .089 & 4.809 .236 & 16.528 .004 & 10.311 .331 & 17.285 .061 & 18.385 .039 \\
\hline CRT TV Subtotal & $\mathbf{6 2 . 6 0 8 . 0 2 3}$ & $\mathbf{6 2 . 4 5 2 . 0 9 7}$ & $\mathbf{7 2 . 1 6 6 . 6 2 8}$ & $\mathbf{2 6 . 5 5 3 . 9 6 5}$ & $\mathbf{4 2 . 8 3 5 . 8 5 4}$ & $\mathbf{4 0 . 7 3 7 . 0 8 4}$ \\
\hline CRT TV Other & 2.091 .768 & 1.550 .825 & 5.626 .636 & 3.250 .298 & 3.900 .127 & 3.894 .089 \\
\hline CRT TV Total & $\mathbf{6 4 . 6 9 9 . 7 9 1}$ & $\mathbf{6 4 . 0 0 2 . 9 2 2}$ & $\mathbf{7 7 . 7 9 3 . 2 6 4}$ & $\mathbf{2 9 . 8 0 4 . 2 6 3}$ & $\mathbf{4 6 . 7 3 5 . 9 8 1}$ & $\mathbf{4 4 . 6 3 1 . 1 7 3}$ \\
\hline Combined TV & 1.275 .659 & 2.026 .860 & 586.643 & 237.971 & 1.094 .724 & 1.087 .717 \\
\hline $\begin{array}{l}\text { Video tuners with screen } \\
\text { and others }\end{array}$ & 606.870 & $\mathbf{7 3 8 . 1 9 1}$ & 1.787 .472 & 838.087 & 3.248 .612 & 4.748 .184 \\
\hline Other TV & 26.672 .118 & 46.355 .626 & 98.309 .489 & 66.210 .363 & 39.841 .402 & 68.958 .274 \\
\hline TV TOTAL* & $\mathbf{9 3 . 2 5 4 . 4 3 8}$ & $\mathbf{1 1 3 . 1 2 3 . 5 9 9}$ & $\mathbf{1 7 8 . 4 7 6 . 8 6 8}$ & $\mathbf{9 7 . 0 9 0 . 6 8 4}$ & $\mathbf{9 0 . 9 2 0 . 7 1 9}$ & $\mathbf{1 1 9 . 4 2 5 . 3 4 8}$ \\
\hline $\begin{array}{l}\text { Total Consumer Elec- } \\
\text { tronics }\end{array}$ & $\mathbf{7 2 7 . 8 0 1 . 5 8 5}$ & $\mathbf{8 2 0 . 5 8 6 . 2 9 2}$ & $\mathbf{1 . 1 0 4 . 3 3 3 . 9 6 8}$ & $\mathbf{6 6 0 . 7 9 6 . 0 2 6}$ & $\mathbf{7 1 3 . 9 7 1 . 4 3 6}$ & $\mathbf{9 7 4 . 1 1 7 . 2 4 3}$ \\
\hline
\end{tabular}

\begin{tabular}{|l|r|r|r|r|r|}
\hline & $\mathbf{2 0 0 4}$ & $\mathbf{2 0 0 5}$ & $\mathbf{2 0 0 6}$ & & $\mathbf{2 0 0 7 * *}$ \\
\hline $\begin{array}{l}\text { CRT TV }(\text { screen diago- } \\
\text { nal<42 cm) }\end{array}$ & 4.414 .435 & 1.751 .570 & 531.198 & CRT TV (screen diagonal<42 cm) & 866.122 \\
\hline $\begin{array}{l}\text { CRT TV }(42 \mathrm{~cm}<\text { screen } \\
\text { diagonal }<52 \mathrm{~cm} \text { ) }\end{array}$ & 1.741 .538 & 750.539 & 917.613 & CRT TV $(42 \mathrm{~cm}<$ screen diagonal $<52 \mathrm{~cm})$ & 26.927 \\
\hline $\begin{array}{l}\text { CRT TV }(52 \mathrm{~cm}<\text { screen } \\
\text { diagonal }<72 \mathrm{~cm})\end{array}$ & 10.187 .620 & 12.523 .501 & 5.561 .619 & CRT TV $(52 \mathrm{~cm}<$ screen diagonal $<72 \mathrm{~cm})$ & 4.466 .837 \\
\hline $\begin{array}{l}\text { CRT TV }(\text { screen diago- } \\
\text { nal }>72 \mathrm{~cm})\end{array}$ & 25.558 .285 & 20.786 .425 & 7.800 .834 & CRT TV (screen diagonal $>72 \mathrm{~cm})$ & 2.063 .233 \\
\hline CRT TV Subtotal & $\mathbf{4 1 . 9 0 1 . 8 7 8}$ & $\mathbf{3 5 . 8 1 2 . 0 3 5}$ & $\mathbf{1 4 . 8 1 1 . 2 6 4}$ & CRT TV Subtotal & $\mathbf{7 . 4 2 3 . 1 1 9}$ \\
\hline CRT TV Other & 16.360 .986 & 13.155 .183 & 2.406 .060 & CRT TV Other*** & 58.945 \\
\hline CRT TV Total & $\mathbf{5 8 . 2 6 2 . 8 6 4}$ & $\mathbf{4 8 . 9 6 7 . 2 1 8}$ & $\mathbf{1 7 . 2 1 7 . 3 2 4}$ & CRT TV Total & $\mathbf{7 . 4 8 2 . 0 6 4}$ \\
\hline Combined TV & 1.165 .243 & 837.705 & 500.515 & Plasma TV*** & 56.545 .213 \\
\hline $\begin{array}{l}\text { Video tuners with screen } \\
\text { and others }\end{array}$ & 11.472 .755 & 43.600 .421 & 119.337 .130 & LCD TV & 204.778 .807 \\
\hline Other TV & 122.948 .210 & 128.463 .970 & 133.718 .213 & Other TV & 491.385 .754 \\
\hline TV TOTAL* & $\mathbf{1 9 3 . 8 4 9 . 0 7 2}$ & $\mathbf{2 2 1 . 8 6 9 . 3 1 4}$ & $\mathbf{2 7 0 . 7 7 3 . 1 8 2}$ & TV TOTAL* & $\mathbf{7 6 0 . 1 9 1 . 8 3 8}$ \\
\hline $\begin{array}{l}\text { Total Consumer Elec- } \\
\text { tronics }\end{array}$ & $\mathbf{1 . 4 1 1 . 2 5 2 . 8 9 1}$ & $\mathbf{1 . 7 4 8 . 4 2 6 . 4 0 2}$ & $\mathbf{1 . 6 8 4 . 2 3 5 . 7 7 1}$ & Total Consumer Electronics & $\mathbf{2 . 2 1 6 . 6 8 3 . 4 3 5}$ \\
\hline
\end{tabular}

* The customs tariff position number " $85-28$ " includes besides television sets, teleprojectors, color video projectors, and satellite television receivers. Since the latter constitutes only a small segment within the category, they were included in "Other TV" and hence 'TV TOTAL".

** The 2007 figures are provisional.

*** According to the legislation pertaining to data confidentiality, certain figures were not released.

Source: TÜİK, 2008b. 
Table 11: Mean Unit Prices of TV Import Items, 1998-2003

\begin{tabular}{|c|c|c|c|c|c|c|c|c|c|}
\hline IMPORT ITEM & $\begin{array}{r}\text { Amount } \\
\text { (Units) }\end{array}$ & $\begin{array}{l}\text { Value } \\
\text { (USD) }\end{array}$ & $\begin{array}{r}\text { Mean Unit } \\
\text { Price(USD) }\end{array}$ & $\begin{array}{r}\text { Amount } \\
\text { (Units) }\end{array}$ & Value (USD) & $\begin{array}{r}\text { Mean Unit } \\
\text { Price(USD) }\end{array}$ & $\begin{array}{r}\text { Amount } \\
\text { (Units) }\end{array}$ & $\begin{array}{r}\text { Value } \\
\text { (USD) }\end{array}$ & $\begin{array}{l}\text { Mean Unit } \\
\text { Price(USD) }\end{array}$ \\
\hline & 1998 & 1998 & 1998 & 1999 & 1999 & 1999 & 2000 & 2000 & 2000 \\
\hline $\begin{array}{l}\text { CRT TV (screen } \\
\text { diagonal< } 42 \mathrm{~cm} \text { ) }\end{array}$ & 118.008 & 14.930 .651 & 126,52 & 230.252 & 16.384 .207 & 71,16 & 193.538 & 13.983 .065 & 72,25 \\
\hline $\begin{array}{l}\text { CRT TV }(42 \\
\mathrm{cm}<\text { screen } \\
\text { diagonal }<52 \mathrm{~cm})\end{array}$ & 83.938 & 15.933 .906 & 189,83 & 67.039 & 10.216 .121 & 152,39 & 68.029 & 7.910 .909 & 116,29 \\
\hline $\begin{array}{l}\text { CRT TV }(52 \\
\mathrm{cm}<\text { screen } \\
\text { diagonal }<72 \mathrm{~cm})\end{array}$ & 97.719 & 26.830 .377 & 274,57 & 137.014 & 31.042 .533 & 226,56 & 186.605 & 33.744 .650 & 180,83 \\
\hline $\begin{array}{l}\text { CRT TV (screen } \\
\text { diagonal }>72 \mathrm{~cm} \text { ) }\end{array}$ & 9.953 & 4.913 .089 & 493,63 & 10.501 & 4.809 .236 & 457,98 & 38.514 & 16.528 .004 & 429,14 \\
\hline CRT TV Subtotal & 309.618 & 62.608 .023 & 202,21 & 444.806 & 62.452 .097 & 140,40 & 486.686 & 72.166 .628 & 148,28 \\
\hline CRT TV Other & 2.894 & 2.091 .768 & 722,79 & 2.036 & 1.550 .825 & 761,70 & 8.545 & 5.626 .636 & 658,47 \\
\hline CRT TV Total & 312.512 & 64.699.791 & 207,03 & 446.842 & 64.002 .922 & 143,23 & 495.231 & 77.793 .264 & 157,08 \\
\hline Combined TV & 2.757 & 1.275 .659 & 462,70 & 2.152 & 2.026 .860 & 941,85 & 5.239 & 586.643 & 111,98 \\
\hline $\begin{array}{l}\text { Video tuners with } \\
\text { screen, etc. }\end{array}$ & 1.500 & 606.870 & 404,58 & 603 & 738.191 & $1.224,20$ & 3.069 & 1.787 .472 & 582,43 \\
\hline Other TV & 440.270 & 26.672 .118 & 60,58 & 607.971 & 46.355 .626 & 76,25 & 873.243 & 98.309 .489 & 112,58 \\
\hline TV TOTAL* & 757.039 & 93.254.438 & 123,18 & 1.057 .568 & 113.123 .599 & 106,97 & 1.376 .782 & 178.476 .868 & 129,63 \\
\hline $\begin{array}{l}\text { Total Consumer } \\
\text { Electronics }\end{array}$ & 8.253.458 & 727.801.585 & & 8.347 .346 & 820.586 .292 & & 10.582 .498 & 1.104 .333 .968 & \\
\hline
\end{tabular}

\begin{tabular}{|c|c|c|c|c|c|c|c|c|c|}
\hline IMPORT ITEM & $\begin{array}{r}\text { Amount } \\
\text { (Units) }\end{array}$ & Value (USD) & $\begin{array}{l}\text { Mean Unit } \\
\text { Price(USD) }\end{array}$ & $\begin{array}{r}\text { Amount } \\
\text { (Units) }\end{array}$ & Value (USD) & $\begin{array}{r}\text { Mean Unit } \\
\text { Price(USD) }\end{array}$ & $\begin{array}{r}\text { Amount } \\
\text { (Units) }\end{array}$ & Value (USD) & $\begin{array}{l}\text { Mean Unit } \\
\text { Price(USD) }\end{array}$ \\
\hline & 2001 & 2001 & 2001 & 2002 & 2002 & 2002 & 2003 & 2003 & 2003 \\
\hline $\begin{array}{l}\text { CRT TV (screen } \\
\text { diagonal< } 42 \mathrm{~cm} \text { ) }\end{array}$ & 64.252 & 4.765 .841 & 74,17 & 118.496 & 5.170 .565 & 43,63 & 228.850 & 8.844 .754 & 38,65 \\
\hline $\begin{array}{l}\text { CRT TV }(42 \\
\mathrm{cm}<\text { screen } \\
\text { diagonal }<52 \mathrm{~cm})\end{array}$ & 23.928 & 2.525 .023 & 105,53 & 16.831 & 1.513 .993 & 89,95 & 10.591 & 1.076 .977 & 101,69 \\
\hline $\begin{array}{l}\text { CRT TV }(52 \\
\mathrm{cm}<\text { screen } \\
\text { diagonal }<72 \mathrm{~cm} \text { ) }\end{array}$ & 50.948 & 8.951 .770 & 175,70 & 127.583 & 18.866 .235 & 147,87 & 112.361 & 12.430 .314 & 110,63 \\
\hline $\begin{array}{l}\text { CRT TV (screen } \\
\text { diagonal }>72 \mathrm{~cm} \text { ) }\end{array}$ & 25.696 & 10.311 .331 & 401,28 & 45.675 & 17.285 .061 & 378,44 & 57.180 & 18.385 .039 & 321,53 \\
\hline CRT TV Subtotal & 164.824 & 26.553.965 & 161,10 & 308.585 & 42.835 .854 & 138,81 & 408.982 & 40.737.084 & 99,61 \\
\hline CRT TV Other & 3.274 & 3.250 .298 & 992,76 & 5.015 & 3.900 .127 & 777,69 & 7.131 & 3.894 .089 & 546,08 \\
\hline CRT TV TOTAL & 168.098 & 29.804 .263 & 177,30 & 313.600 & 46.735.981 & 149,03 & 416.113 & 44.631.173 & 107,26 \\
\hline COMBINED TV & 710 & 237.971 & 335,17 & 4.585 & 1.094 .724 & 238,76 & 5.160 & 1.087 .717 & 210,80 \\
\hline $\begin{array}{l}\text { VIDEO TUN- } \\
\text { ERS WITH } \\
\text { SCREEN and } \\
\text { others }\end{array}$ & 5.203 & 838.087 & 161,08 & 4.613 & 3.248 .612 & 704,23 & 9.297 & 4.748 .184 & 510,72 \\
\hline Other TV & 486.149 & 66.210 .363 & 136,19 & 380.596 & 39.841 .402 & 104,68 & 779.093 & 68.958 .274 & 88,51 \\
\hline TV Total* & 660.160 & 97.090 .684 & 147,07 & 703.394 & 90.920 .719 & 129,26 & 1.209 .663 & 119.425 .348 & 98,73 \\
\hline $\begin{array}{l}\text { Total Consumer } \\
\text { Electronics }\end{array}$ & 4.793.957 & 660.796 .026 & & 88.696 .142 & 713.971.436 & & 89.175.735 & 974.117.243 & \\
\hline
\end{tabular}

* The customs tariff position number " $85-28$ " includes besides television sets, teleprojectors, color video projectors, and satellite television receivers. Since the latter constitutes only a small segment within the category, they were included in "Other TV" and hence "TV TOTAL".

** The 2007 figures are provisional.

*** According to the legislation pertaining to data confidentiality, certain figures were not released.

Source: TÜIK, 2008 b. 
Table 12: Mean Unit Prices of TV Import Items, 2004-2007

\begin{tabular}{|c|c|c|c|c|c|c|c|c|c|}
\hline $\begin{array}{l}\text { IMPORT } \\
\text { ITEM }\end{array}$ & $\begin{array}{r}\text { Amount } \\
\text { (Units) }\end{array}$ & Value (USD) & $\begin{array}{l}\text { Mean Unit } \\
\text { Price(USD) }\end{array}$ & $\begin{array}{r}\text { Amount } \\
\text { (Units) }\end{array}$ & Value (USD) & $\begin{array}{r}\text { Mean Unit } \\
\text { Price } \\
\text { (USD) }\end{array}$ & $\begin{array}{r}\text { Amount } \\
\text { (Units) }\end{array}$ & Value (USD) & $\begin{array}{l}\text { Mean Unit } \\
\text { Price(USD) }\end{array}$ \\
\hline & 2004 & 2004 & 2004 & 2005 & 2005 & 2005 & 2006 & 2006 & 2006 \\
\hline $\begin{array}{l}\text { CRT TV (screen } \\
\text { diagonal }<42 \mathrm{~cm} \text { ) }\end{array}$ & 93.205 & 4.414 .435 & 47,36 & 22.840 & 1.751 .570 & 76,69 & 4.185 & 531.198 & 126,93 \\
\hline $\begin{array}{l}\text { CRT TV (42 } \\
\mathrm{cm}<\text { screen } \\
\text { diagonal }<52 \\
\mathrm{~cm})\end{array}$ & 6.988 & 1.741 .538 & 249,22 & 4.060 & 750.539 & 184,86 & 1.930 & 917.613 & 475,45 \\
\hline $\begin{array}{l}\text { CRT TV }(52 \\
\text { cm<screen } \\
\text { diagonal }<72 \\
\mathrm{~cm})\end{array}$ & 70.812 & 10.187 .620 & 143,87 & 65.226 & 12.523 .501 & 192,00 & 29.546 & 5.561 .619 & 188,24 \\
\hline $\begin{array}{l}\text { CRT TV (screen } \\
\text { diagonal >72 } \\
\mathrm{cm} \text { ) }\end{array}$ & 86.205 & 25.558 .285 & 296,48 & 56.574 & 20.786 .425 & 367,42 & 21.867 & 7.800 .834 & 356,74 \\
\hline $\begin{array}{l}\text { CRT TV } \\
\text { Subtotal }\end{array}$ & 257.210 & 41.901.878 & 162,91 & 148.700 & 35.812.035 & 240,83 & 57.528 & 14.811 .264 & 257,46 \\
\hline CRT TV Other & 22.981 & 16.360 .986 & 711,94 & 17.442 & 13.155 .183 & 754,22 & 6.926 & 2.406 .060 & 347,40 \\
\hline CRT TV Total & 280.191 & 58.262 .864 & 207,94 & 166.142 & 48.967.218 & 294,73 & 64.454 & 17.217 .324 & 267,13 \\
\hline Combined TV & 4.068 & 1.165 .243 & 286,44 & 3.912 & 837.705 & 214,14 & 3.381 & 500.515 & 148,04 \\
\hline $\begin{array}{l}\text { Video tuners } \\
\text { with screen, etc. }\end{array}$ & 24.051 & 11.472 .755 & 477,02 & 67.968 & 43.600 .421 & 641,48 & 140.890 & 119.337 .130 & 847,02 \\
\hline Other TV & 2.035 .953 & 122.948 .210 & 60,39 & 1.368 .152 & 128.463 .970 & 93,90 & 476.109 & 133.718 .213 & 280,86 \\
\hline TV TOTAL* & 2.344.263 & 193.849.072 & 82,69 & 1.606 .174 & 221.869.314 & 138,14 & 684.834 & 270.773.182 & 395,39 \\
\hline $\begin{array}{l}\text { Total Consumer } \\
\text { Electronics }\end{array}$ & 164.265 .132 & 1.411 .252 .891 & & 119.422 .618 & 1.748 .426 .402 & & 85.504 .889 & 1.684 .235 .771 & \\
\hline
\end{tabular}

\begin{tabular}{|l|r|r|r|}
\hline Import Item & Amount (Units) & Value (USD) & Mean Unit Price (USD) \\
\hline & $\mathbf{2 0 0 7 * * *}$ & $\mathbf{2 0 0 7 * *}$ & $\mathbf{2 0 0 7 * *}$ \\
\hline CRT TV $($ screen diagonal $42 \mathrm{~cm})$ & 8.239 & 866.122 & 105,12 \\
\hline CRT TV $(42 \mathrm{~cm}<$ screen diagonal $<52 \mathrm{~cm})$ & 98 & 26.927 & 274,77 \\
\hline CRT TV $(52 \mathrm{~cm}<$ screen diagonal $<72 \mathrm{~cm})$ & 17.321 & 4.466 .837 & 257,89 \\
\hline CRT TV $($ screen diagonal $>72 \mathrm{~cm})$ & 6.984 & 2.063 .233 & 295,42 \\
\hline CRT TV Subtotal & $\mathbf{3 2 . 6 4 2}$ & $\mathbf{7 . 4 2 3 . 1 1 9}$ & 227,41 \\
\hline CRT TV Other** & 96 & 58.945 & 614,01 \\
\hline CRT TV Total & $\mathbf{3 2 . 7 3 8}$ & $\mathbf{7 . 4 8 2 . 0 6 4}$ & 228,54 \\
\hline Plasma TV*** & 49.577 & 56.545 .213 & $1.140,55$ \\
\hline LCD TV & 266.647 & 204.778 .807 & 767,98 \\
\hline Other TV & 3.278 .167 & 491.385 .754 & 149,90 \\
\hline TV TOTAL* & $\mathbf{3 . 6 2 7 . 1 2 9}$ & $\mathbf{7 6 0 . 1 9 1 . 8 3 8}$ & 209,59 \\
\hline Total Consumer Electronics & $\mathbf{6 0 1 . 4 7 8 . 5 1 4}$ & $\mathbf{2 . 2 1 6 . 6 8 3 . 4 3 5}$ & \\
\hline
\end{tabular}

* The customs tariff position number "85-28" includes besides television sets, teleprojectors, color video projectors, and satellite television receivers. Since the latter constitutes only a small segment within the category, they were included in "Other TV" and hence "TV TOTAL".

** The 2007 figures are provisional.

*** According to the legislation pertaining to data confidentiality, certain figures were not released.

Source: TÜIK, 2008b. 


\section{The Turkish Domestic Market for Television Sets}

The volume of home market sales for television sets is estimated to be around 2.5 million sets for 2007 including both cathode-ray tube televisions and LCD televisions, which together account for around 413 million US dollars (Turkish Ministry of Industry and Trade, 2008).

The cathode-ray tube television market in Turkey decreases by $50 \%$ yearly, and the LCD market grows by $100 \%$ (ibid.). A recent consumer research indicates that the growth trend for LCD and plasma televisions is likely to continue unabated. In the country-wide study conducted by GfK Turkey ("Türklerin," 2008), 23\% of the consumers had plasma TV within the top five items they intended to buy; the comparable figure for LCD TV was $14 \%$.

As television set penetration in Turkey is estimated at 97\% for 2006 (World Bank, 2008), it can be inferred that demand for television sets is largely driven by renewal and replacement purchases. Television set lifespan is considered to be 12 years on average (Çakýr, 2004: 11), but, renewal demand for new models with added features before the old TV set completes its lifespan is estimated to constitute about one-third of all renewal purchases per year, i.e., those spurred by new home purchases, opening up of a new business, and purchases of secondary sets in addition to the primary one (Çakir, 2004: 11).

The main brands in the home market are Beko with a 36\% share, Vestel, with a 35\% share, and Profilo with a 9\% share for 2007 (Turkish Ministry of Industry and Trade, 2008). Vestel, Beko and Profilo also account for $95 \%$ of the current production capacity for television manufacturing (ibid.). Around $20 \%$ of home market sales are composed of imported brands (ibid.), which include Philips, Pioneer, Panasonic, Samsung, Sony, LG, and Toshiba (according to information obtained from various retailers). When it comes to LCD televisions, around 50\% are imported. Of the 678.000 LCD TVs sold in Turkey in 2007, imported brands accounted for 44\% (or, 300,000 sets) (Turkish Ministry of Industry and Trade).

\section{Table 13: Major Manufacturers of Television Sets in Turkey} MAIN COMPANIES IN TURKEY'S CONSUMER ELECTRONICS INDUSTRY

\begin{tabular}{|l|r|r|r|r|r|r|r|}
\hline & $\begin{array}{r}\text { Proportion } \\
\text { of exports in } \\
\text { sales* } \% \text { ) }\end{array}$ & $\begin{array}{r}\text { Sales from } \\
\text { production } \\
\text { net* } \\
\text { (YTL) }\end{array}$ & $\begin{array}{r}\text { Export amount* } \\
(\$)\end{array}$ & $\begin{array}{r}\text { Total TV } \\
\text { sales (units) } \\
* *\end{array}$ & $\begin{array}{r}\text { Total TV } \\
\text { exports } \\
\text { (units)** }\end{array}$ & $\begin{array}{r}\text { Home mar- } \\
\text { ket TV sales } \\
\text { (units)*** }\end{array}$ & $\begin{array}{r}\text { Company's } \\
\text { share in the } \\
\text { Turkish } \\
\text { market for } \\
\text { TV sales } \\
\text { volume }\end{array}$ \\
\hline VESTEL & $89 \%$ & 2.395 .395 .531 & 1.688 .850 .000 & 8.776 .324 & 7.372 .112 & 1.404 .212 & $35 \%$ \\
\hline $\begin{array}{l}\text { BEKO ELEKTRO- } \\
\text { NIK (now GRUNDIG } \\
\text { ELEKTRONIK) }\end{array}$ & $73 \%$ & 1.063 .270 .906 & 557.947 .000 & 3.435 .707 & 2.508 .066 & 927.641 & $36 \%$ \\
\hline PROFILO TELRA & $41 \%$ & 169.475 .191 & 69.376 .000 & & & & \\
\hline
\end{tabular}

* Istanbul Chamber of Industry (2008).

** Data is from the companies' annual reports for 2007.

$* * *$ Computed from the figures given in the annual reports.

${ }^{a}$ Turkish Ministry of Industry and Trade (2008). 
It can be seen from the above figures that Vestel and Beko together account for around $90 \%$ of total TV unit exports for 2007.

Vestel is the top manufacturer of consumer electronics products; for instance, $67 \%$ of all televisions produced in Turkey today are manufactured by Vestel (Önkol, 2008). Vestel Electronics joined the Zorlu Group of Companies in 1994. The company manufactures and sells CRT TVs, TFT-LCDs, and monitors. In 2007, the company's market share in Europe was 21\%; in CRT TVs, Vestel is by far the leader in Europe, with 50\% of the market, and in LCD TVs, it has a market share of $11 \%$ (Vestel, 2008: 21). The company's central office is located in Istanbul, and the production plant is in Manisa, Turkey. The company is an OEM/ODM in the European Union, and in Turkey and neighboring countries, it markets its televisions under the Vestel brand name (Vestel, 2008: 20).

The second biggest manufacturer is Arçelik, considered the flagship of Turkey's biggest conglomerate Koç Group of Companies. Arçelik manufactures and sells its televisions under the brand names of Arçelik and Beko. Beko is the electronics arm of Arçelik, and following the recent acquisition of the prestigious German consumer electronics company Grundig in 2007, Beko Elektronik was renamed Grundig Elektronik, which was subsumed entirely under Arçelik in February 2009 (newspaper reports of February 28, 2009).

Profilo, the third biggest manufacturer, began production in 1972 with the manufacturing of blackand-white television sets. The company manufactures Philips-branded television sets in addition to its own brand and Telefunken.

\section{Opportunities and Challenges for the Industry}

The biggest challenge facing Turkey's television set industry today appears to be the need for transition to LCD and plasma TV manufacturing. Eight out of ten television sets sold in Europe today are LCDs (Turkish Ministry of Industry and Trade, 2008), and the shift in consumer trends towards LCD and plasma televisions forces Turkish television producers to invest in new technologies (Tolga and Akýn, 2008). For now, Turkish manufacturers produce LCD TVs using imported parts and run into difficulties obtaining LCD panels (Turkish Ministry of Industry and Trade, 2008).

The research and development costs required by LCD and plasma television set manufacturing are high, and Turkish manufacturers' total R\&D spending is only a fraction of that of their Far Eastern rivals (Please see Table 14).

\section{Table 14: Television Set Manufacturers' Research and Development Spending, 2007}

\begin{tabular}{|l|r|}
\hline Company & $\begin{array}{r}\text { R\&D Spending } \\
\text { (Million US dollars) }\end{array}$ \\
\hline Samsung & 4,600 \\
\hline LG & 4,000 \\
\hline Daewoo & 3,000 \\
\hline Hyundai & 2,000 \\
\hline Turkey & 40 \\
\hline Source: Turkish Ministry of Industry and Trade, 2008. & \\
\hline
\end{tabular}


As it is, the television sets manufactured in Turkey are taxed at 55\% (VAT: 18\%, stamp tax 16\%, special consumption tax 7\%, customs duty 14\%) (Önkol, 2008). With keen price competition, manufacturers complain of low profit margins and indicate the need for government support in $R \& D$ (ibid.).

Some government support came in 2008 in the form of a law to support research and development activities of industry (Legislation No. 5746, passed on Feb. 28, 2008). According to the law, investors will benefit until the year 2024 from a variety of incentives and government support irrespective of their area of activity.

Turkey's television set industry claims to have a highly qualified workforce of 12,300. The Turkish Ministry of Industry and Trade believes that over twenty years of export experience to Europe has resulted in a solid brand management capability and the establishment of a strong distribution and after-sales service network (Turkish Ministry of Industry and Trade, 2008). We have no independent evidence to support this claim.

A research report concluded that Turkish manufacturers also have the capability to manufacture to short schedules. In fact, some of the manufacturers in the industry are held up as examples of flexible manufacturing (Ateş, 2006: 137).

Some of the major television manufacturers in the international arena are forming strategic alliances in Turkey, and there is some indication that a few are moving some of their branded manufacturing operations to Turkey. Since 2004, for instance, Sony televisions are sold at Arçelik retail outlets, and in December of 2008, Grundig Elektronik, an Arçelik subsidiary which received a Sony OEM-Green Partner certificate, will be manufacturing Sony LCD televisions (Grundig Elektronik, 2008).

Although the present export value of the Turkish television industry is not high in global terms, it is possible that with significant $R \& D$ investments (which the new legislation is likely to stimulate), engineering quality, and broad product range, the industry may in a number of years' time substantially increase its share of the global market. We make some further comment on global issues below.

\section{DISCUSSION AND CONCLUSION}

In terms of the validity of the research evidence presented in this preliminary study, we are confident that it is statistically valid. Moreover, the data is longitudinal unlike immediate survey data and therefore presents a useful historical perspective on Turkish international development to the year 2008. We are also confident that we have been able to separate the factual evidence from the judgements of opinion formers in the industry.

However, there are three problems with the data. First, it does not take into account the major world downturn in the global economy that occurred in 2009 and possibly beyond this time. The early industry evidence suggests that this is having an impact on consumer electronics worldwide, including television set manufacture. It may therefore be that the picture presented in this report will change significantly over the year 2009. We will therefore re-visit the data at a later stage and adjust as necessary.

The second problem with the data is that it is eclectic and non-selective. It makes no attempt to develop hypotheses based on international development theories and then test them using the data. This report essentially summarizes the current information on the Turkish television set industry with 
regard to international development. Hence, it is possible that it includes information that will subsequently prove redundant. However, as a first step, we have judged it prudent to present a full picture of the data set. When we develop the research further, we would anticipate starting from a stronger basis in international trade research hypotheses.

The third problem with the data is that it provides only limited information on the reasons why the Turkish industry has developed along its current trajectory. For example, we know that exports have primarily been targeted at Europe but our only evidence on the reasons for this are, at best, anecdotal. The next stage of our research will address this area. But, as stated in the opening paragraphs, this report represents a preliminary study and it remains valid as a picture of the situation to year 2008.

In conclusion, this is only a preliminary examination of the Turkish television set market. The questions we will be exploring in further research will include the reasons why Turkish exports are mostly headed to the European Union within the context of international expansion strategies. Are there elements of cultural distance, administrative distance, economic distance, and in some instances, geographic distance, that are keeping Turkish manufacturers from expanding more into other markets beyond the EU? Why have Turkish exporters chosen particular strategies to export their goods? The answers may have critical implications for Turkish exporters in a variety of markets. 


\section{REFERENCES}

"Grundig Elektronik, Sony LCD televizyon üretecek." (Grundig Elektronik will manufacture Sony LCD televisions). (2008, December 27). Hürriyet, p. 12.

"Türklerin tekno rüyası, plazma TV, cep, laptop." (Plasma TV, cellular phones, and laptops are what consumers dream of). (2008, December 22). Milliyet, p. 6.

Ateş, R. (2006). Şirket Doktoru. İstanbul: Hayat Yayincilik.

Axinn, C. N., Matthyssens, P. (2002) Limits of internationalization theories in an unlimited world. International Marketing Review, 19(5), 436-449.

Barney, J.B. (1991), "Firm resources and sustained competitive advantage", Journal of Management, Vol. 17, pp.99-120.

Bartlett C.A. and Ghoshal S. (1989) Managing Across Borders: The Transnational Solution, Century Business, London.

Beko. (2008) Annual report 2007. Retrieved from www.beko.com.tr in December 2008.

Buckley, P. J. (2002) "Is the international business research agenda running out of steam?" Journal of International Business Studies, 33(2), 365-373.

Çakır, E. (2004). Report on the Turkish Television Industry (ITO Televizyon Sektör Raporu). Istanbul: The Istanbul Chamber of Commerce.

Delios, A. and Henisz, W.J. (2003) Policy uncertainty and the sequence of entry by Japanese firms 1980-1998, Journal of International Business Studies, Vol 34, No 3, pp 227-241

Douglas S. and Wind Y. (1987) The Myth of Globalization, Columbia Journal of World Business, Volume 22, No.4, Winter, pp 19-29.

Doz, Y.L., Santos, J and Williamson, P (2001) From Global to Metanational: how companies win in the global economy, Harvard Business School Press, Boston, MA

DPT. (2007). Elektronik ve Elektrikli Makinalar Sanayii: Özel İhtisas Komisyonu Raporu. (Electronics and Electrical Machinery Industry: Report of the Special Expert Commission.) Ankara: State Planning Organization of Turkey.

DTM. (2006). Elektrik-Elektronik Sektörü (Electrical and Electronics Industry). Ankara: Undersecretary of Foreign Trade. Retrieved February 2009 from http://www.dtm.gov.tr/dtadmin/upload/ihr/ otomotivelektrikdb/elektrik_elektronik_sektoru.doc.

DuBravac, S. G. (2007). The US Television Set Market. Business Economics 42, no. 3 (July).

Dunning J.H. (1995) The Globalization of Business, Routledge, London and NY, Ch 5.

Dunning, J.H. and Lundan, S. M. (2008) Multinational Enterprises and the Global Economy, $2^{\text {nd }}$ Edition, Edward Elgar, Cheltenham UK, Tables 2.5 and 2.6

Eurostat. (2007). Panorama of European Union Trade: Data 1999-2006. Retrieved October 2008 from http://ec.europa.eu/eurostat.

Forsgren, M (2002) The concept of learning in the internationalization process model: a critical re- 
view, International Business Review, Vol 11, No 3, pp 257-277

Ghemawat, P. (2001) Distance still matters, Harvard Business Review, September, Vol 79, Issue 8.

Ghemawat, P. (2003) Semiglobalization and international business strategy, Journal of International Business Studies, Vol 34, pp 138-152.

Ghemawat, P. and Ghadar, F. (2000) The dubious logic of global megamergers, Harvard Business Review, July August, Vol 78, Issue 4.

Ghoshal, S. (1987) Global Strategy: an Organising Framework, Strategic Management Journal, Vol 8, pp 424-440.

Istanbul Chamber of Industry. (2001). The Electronics and Telecommunications Sector (Elektronik ve Telekomünikasyon Sektörü). Istanbul: Istanbul Chamber of Industry, publication no. 2001/17.

Istanbul Chamber of Industry. (2008). Istanbul Chamber of Industry Magazine Special Issue: Turkey's 500 Largest Industrial Companies. Istanbul: Istanbul Chamber of Industry, v. 43, no. 509 (August).

Johanson, J. and Vahlne, E. (1977) The internationalization process of the firm - a model of knowledge development and increasing market commitments, Journal of International Business Studies, Vol 8, No 1, pp 23-32.

Karabatı, S. and Tan, B. (2005). Vestel Electronics: Transition into the leading European TV manufacturer, Koç University Graduate School of Business, Istanbul, Turkey, Case Number 605-015-1.

Lynch, R. (2008) Strategic Management fifth edition, Pearson Education, Harlow. Chapter 4.

Önkol, B. (2008, 15 September). "Temel sorun üretmemek değil, değerli YTL ve düpen fiyatlar" (The main problem of the Turkish television industry is not production-related, but the valuable lira and declining prices). Milliyet Business: 7.

Peng, M. W. (2005) Perspectives from China strategy to global strategy. Asia pacific Journal of Management, 22, 123-141.

Peng, M.W. and Heath, P. S. (1996): 'The growth of the firm in planned economies in transition: institutions, organisations and strategic choice', Academy of Management Review, 21:2 pp.492-528

Rugman, A.M. and Verbeke, A. (2003a) Regional Multinationals: the Location-Bound Drivers of Global Strategy, In: Burkinshaw, J., Ghoshal, S., Markides, C., Stopford, J., Yip, G., (eds) The Future of the Multinational Company, Wiley, Chichester.

Rugman, A.M. and Verbeke, A. (2003b) Extending the theory of the multinational enterprise: internationalization and strategic management perspectives, Journal of International Business Studies, Vol 34, pp 125-137.

Tallman, S. (2001) Global Strategic Management. In: Hit, M.A., Freeman, R.E. and Harrison, J.S. (eds) The Blackwell Handbook of Strategic Management, Blackwell, Oxford.

Taylan, T. and Sarı, A. (2008). "Electronics industry”, Export Promotion Center of Turkey.

TÜİK. (2008a). Television production output, 1983-2007. [Data file]. Ankara: The Turkish Statistics Institute. 
TÜIK. (2008b). Foreign trade statistics related to selected items between the years of 1998-2007. [Data files] Ankara: The Turkish Statistics Institute.

TÜIKK. (2009). Annual production and sales figures related to selected items between the years of 1997-2008. [Data files] Ankara: The Turkish Statistics Institute.

Turkish Ministry of Industry and Trade. (2008). "Situation analysis of the television industry," Presentation (June), Ankara: The Turkish Ministry of Industry and Trade.

UNCTAD (2008) Handbook of Statistics Table 1.1 Value shares of merchandise exports and imports Usta, H. (2002). Report on the Turkish Brown Goods Industry (ITO Kahverengi Epya Sektör Profil Araştırmasi). Istanbul: The Istanbul Chamber of Commerce.

Vestel. (2008) Annual report 2007. Retrieved from www.vestel.com.tr in December 2008.

Werner, S. (2002) Recent developments in international management research: A review of 20 top management journals. Journal of Management, 28 (3), 277-305.

World Bank (2008). Retrieved from www.worldbank.org in October 2008. 